\title{
Age-associated changes in expression of small, noncoding RNAs, including microRNAs, in C. elegans
}

\author{
MASAOMI KATO, ${ }^{1}$ XIAOWEI CHEN, ${ }^{1,2}$ SACHI INUKAI, ${ }^{1}$ HONGYU ZHAO, ${ }^{2,3,4}$ and FRANK J. SLACK ${ }^{1,5}$ \\ ${ }^{1}$ Department of Molecular, Cellular and Developmental Biology, Yale University, New Haven, Connecticut 06520, USA \\ ${ }^{2}$ Program in Computational Biology and Bioinformatics, Yale University School of Medicine, New Haven, Connecticut 06520, USA \\ ${ }^{3}$ Department of Epidemiology and Public Health, Yale University School of Medicine, New Haven, Connecticut 06520, USA \\ ${ }^{4}$ Department of Genetics, Yale University School of Medicine, New Haven, Connecticut 06520, USA
}

\begin{abstract}
Small, noncoding RNAs (sncRNAs), including microRNAs (miRNAs), impact diverse biological events through the control of gene expression and genome stability. However, the role of these sncRNAs in aging remains largely unknown. To understand the contribution of sncRNAs to the aging process, we performed small RNA profiling by deep-sequencing over the course of Caenorhabditis elegans (C. elegans) aging. Many small RNAs, including a significant number of miRNAs, change their expression during aging in $C$. elegans. Further studies of miRNA expression changes under conditions that modify lifespan demonstrate the tight control of their expression during aging. Adult-specific loss of argonaute-like gene-1 (alg-1) activity, which is necessary for miRNA maturation and function, resulted in an abnormal lifespan, suggesting that miRNAs are, indeed, required in adulthood for normal aging. miRNA target prediction algorithms combined with transcriptome data and pathway enrichment analysis revealed likely targets of these age-associated miRNAs with known roles in aging, such as mitochondrial metabolism. Furthermore, a computational analysis of our deep-sequencing data identified additional age-associated sncRNAs, including miRNA star strands, novel miRNA candidates, and endo-siRNA sequences. We also show an increase of specific transfer RNA (tRNA) fragments during aging, which are known to be induced in response to stress in several organisms. This study suggests that sncRNAs including miRNAs contribute to lifespan regulation in $C$. elegans, and indicates new connections between aging, stress responses, and the small RNA world.
\end{abstract}

Keywords: aging; miRNA; noncoding RNA; C. elegans; modEncode

\section{INTRODUCTION}

Aging is defined as the time-dependent degenerative changes that occur in tissues, cells, and in molecules such as DNA. For example, loss of muscle mass and strength usually begins at the middle of adulthood and proceeds further during the later stages of life in diverse animal species, including the nematode Caenorhabditis elegans (C. elegans) (Herndon et al. 2002; Johnston et al. 2008). These physiological alterations are correlated with a decline in motility and closely resemble sarcopenia in humans (Herndon et al. 2002). Also, progressive accumulation of a fluorescent pigment, lipofuscin, is observed in aging tissues as a result of the oxidative degradation and autophagocytosis of cellular components (Garigan et al. 2002). Its ubiquitous occurrence in a

\footnotetext{
${ }^{5}$ Corresponding author.

E-mail frank.slack@yale.edu.

Article published online ahead of print. Article and publication date are at http://www.rnajournal.org/cgi/doi/10.1261/rna.2714411.
}

wide variety of organisms makes it a universal biomarker for aging. Additionally, it is known that aspects of nuclear architecture, such as nuclear shape and peripheral heterochromatin, are disorganized during aging in C. elegans and that such features are often observed in premature aging syndromes, including Hutchinson-Gilford Progeria Syndrome (HGPS) in humans as well (Mattout et al. 2006).

It had been thought that age-related functional declines were passive and not subject to regulation. Yet, it has been demonstrated in C. elegans that mutations in single genes can cause a delay in the aging process and extend lifespan. age-1 and daf-2, both of which encode components in an insulin signaling cascade, cause more than a twofold increase in lifespan when either activity is reduced (Klass 1983; Friedman and Johnson 1988; Kenyon et al. 1993; Morris et al. 1996). Following these pioneering genetic studies in C. elegans, it has been shown that these longevity genes are highly conserved in yeast, flies, and mice and that they also regulate lifespan through the control of, for example, metabolism and the stress response, across phyla (Antebi 2007; 
Kenyon 2010). These findings suggest that lifespan is, at least in part, a genetically determined biological event and that changes in gene expression may underlie at least some of the aging processes, including metabolic changes.

Gene expression profiling during aging can provide insight into the molecular basis of the aging process. C. elegans provides a genetically tractable model for this, with its relatively short lifespan and plentiful genetic resources. Studies of gene expression changes during aging in C. elegans have provided clues to understanding mechanisms of age-related phenomena. For example, the study of Lund et al. (2002) demonstrated an increase in expression of transposable element-derived transcripts during aging, pointing out the potential of increased genome instability or uncontrolled, unfavorable transcriptional activation with age. Another study showed that many genes encoding heat shock proteins, which are stress-resistance genes activated by stress, rise in their expression at the beginning of adulthood and then decrease toward later stages of aging (Golden and Melov 2004; McCarroll et al. 2004). Since heat shock proteins serve as molecular chaperones in protein homeostasis (Morimoto 2008), their decrease in aged animals may cause an increased level of unfolded proteins, possibly leading to increased toxicity and stress, finally causing impaired cellular function and organismal death. These studies support the idea that alteration of gene activity might serve as a biological clock influencing lifespan.

Notably, small noncoding RNAs (sncRNAs), especially microRNAs (miRNAs), have a great impact on the control of gene expression through their ability to regulate hundreds of target genes (Stefani and Slack 2008). miRNAs negatively regulate gene expression at the post-transcriptional level via perfect or partial sequence complementarity to mRNAs of their target genes. For example, a founding miRNA, lin-4, accumulates in the early larval stage of C. elegans development and suppresses the expression of its target gene lin-14 to initiate a transition in developmental stage (Lee et al. 1993). Importantly, in a previous study, we found that the lin-4 miRNA and its target lin-14 are also necessary for normal lifespan in C. elegans; lin-4 loss-of-function mutants and gain-of-function mutants of its target lin-14 (due to loss of a lin- 4 binding site in its $3^{\prime}$ untranslated region) showed a short lifespan, while overexpression of lin-4 miRNA and loss-of-function mutants of lin-14 resulted in a longer lifespan (Boehm and Slack 2005), suggesting that miRNA and miRNA-mediated negative regulation of gene expression influence longevity. Subsequent miRNA microarray analysis showed that many mature miRNAs change their expression during aging in C. elegans (Ibanez-Ventoso et al. 2006). In addition to miRNAs, additional new classes of sncRNAs identified in recent studies (Ruby et al. 2006; Batista et al. 2008; Claycomb et al. 2009; Gu et al. 2009; Stoeckius et al. 2009), such as 21U-RNAs/piRNAs and 22G-RNAs (endo-siRNAs), might also have important roles in aging through the maintenance of genome stability and genome surveillance, but the dynamics of these sncRNA species through aging have not been examined.

Here, we show, using deep-sequencing technology, that a significant number of sncRNAs, including miRNAs, change their expression during aging in C. elegans. In addition, adultspecific knockdown of argonaute-like gene-1 (alg-1), which is required for miRNA processing and function, resulted in a shorter lifespan, supporting our hypothesis that miRNAs have important roles in normal lifespan regulation. Also, predicted targets of age-associated miRNAs have known roles in aging processes, including mitochondrial respiration and protein homeostasis. We also report the identification of novel miRNA candidates, miRNA star molecules, and other age-associated sncRNAs, including transfer RNA (tRNA) cleavage products, which are known to be induced in response to stress in several organisms (Thompson and Parker 2009a). This study uncovers potential roles for miRNAs and other sncRNAs in aging and age-related events, and we propose that these RNAs contribute to lifespan regulation in C. elegans.

\section{RESULTS}

In order to reveal the contributions of sncRNAs, especially miRNAs, to aging in C. elegans, we first examined their expression changes during aging, using deep-sequencing with Solexa technology. In this study, we used spe-9(hc88), a temperature-sensitive sterile mutant, to prevent contamination of RNAs from aging animals with those of developing embryos. Although spe-9(hc88) mutant animals cannot produce progeny at the restrictive temperature due to a defect in spermatogenesis (Singson et al. 1998), they still have a lifespan similar to wild-type N2 animals (Fabian and Johnson 1994). We first confirmed their normal lifespan at the nonpermissive temperature, $23^{\circ} \mathrm{C}$ (Fig. 1A), and prepared cDNA libraries for small RNAs at four different time points during adulthood: Day 0, Day 5, Day 8, and Day 12 (Day X represented in this study is defined as days post-L4 [the fourth larval stage] molt). As shown in Figure 1A, these times represent key inflection points in the survival curve. The approximate survival rate of spe-9(hc88) mutants was $100 \%,>90 \%, 50 \%$, and $<10 \%$ at Day 0 , Day 5, Day 8 , and Day 12, respectively. We also produced additional cDNA libraries from RNA samples that were collected from independently grown Day 0 and Day 8 spe-9(hc88) mutants in order to test the reproducibility of the initial experiment.

More than 5 million sequence reads that match to the $C$. elegans genome were obtained from each library (Supplemental Table S1). Of those, $\sim 70 \%$ were known, annotated mature miRNA sequences, $\sim 1.5 \%$ of reads perfectly matched $21 \mathrm{U}$-RNAs/piRNAs, $\sim 15 \%$ mapped to a part of other known noncoding RNA fragments such as tRNAs, and the remaining $15 \%$ did not map to any annotated regions and were categorized into a fraction of "other reads" (Fig. 1B; Supplemental Table S1). This fraction includes miRNA- 
A

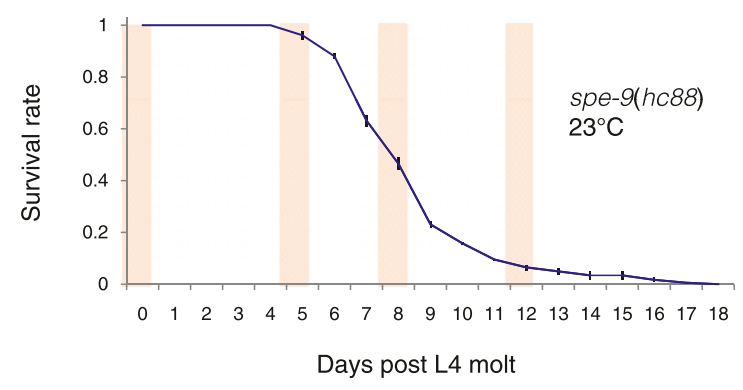

B

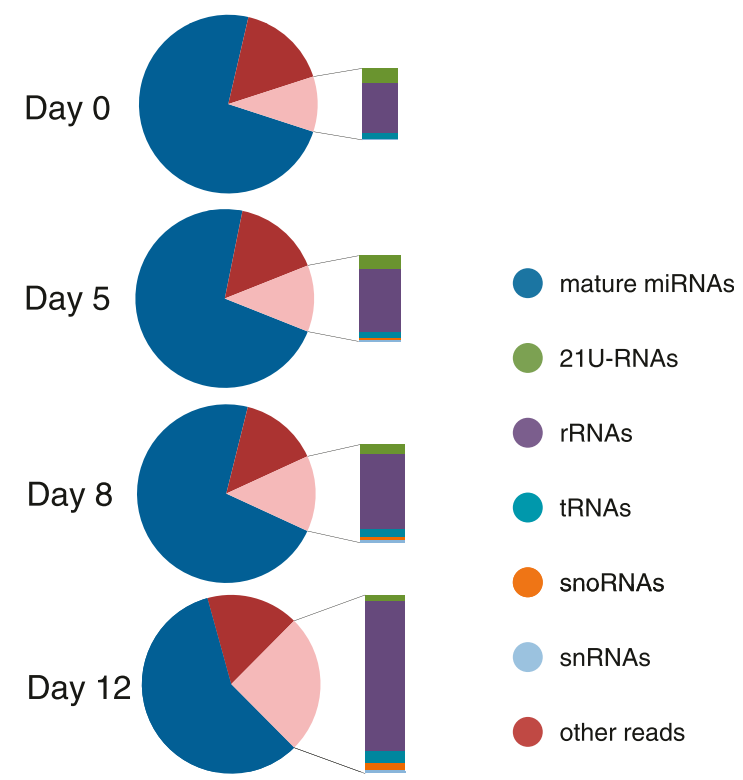

FIGURE 1. Lifespan of spe-9(hc88) mutant animals and summary of deep-sequencing reads. $(A)$ Animals were raised and cultured at $23^{\circ} \mathrm{C}$ in the lifespan assay. Mean adult lifespan was $8.62+/-0.05 \mathrm{~d}$. Detailed results of the lifespan assay are shown in Supplemental Table S4. Error bars represent standard error (SE) calculated from triplicates. RNAs were purified at Day 0, Day 5, Day 8, and Day 12 post-L4 molt as highlighted and used for library preparation for Solexa deep-sequencing experiments. (B) Proportion of each noncoding RNA species, including miRNAs, 21U-RNAs/piRNAs, and tRNAs, were analyzed in each aging sample. Details are shown in Supplemental Table S1.

associated reads such as miRNA "star" strands, and novel miRNA candidates and possible novel sncRNAs (described below). We also found that the total levels of both miRNAs and 21U-RNAs/piRNAs exhibited a gradual decrease during aging, while other small RNA fragments derived from tRNAs, ribosomal RNAs (rRNAs), small nucleolar RNAs (snoRNAs), and small nuclear RNAs (snRNAs) showed a gradual but constant increase in accumulation during aging (Fig. 1B; Supplemental Table S1) (details described below).

\section{A significant number of mature miRNAs change their expression during aging}

As mentioned, most of the aligned deep-sequencing reads mapped to known, annotated mature miRNAs. We could detect reads from 149 of 174 annotated mature miRNAs in our libraries (based on miRBase release 14) (Kozomara and Griffiths-Jones 2011; Supplemental Tables S2, S3). Of the known miRNAs we could not detect, some may not actually encode miRNAs, or their mature strands may have been mistakenly annotated. For example, we did not clone sequences corresponding to the annotated miR-356 mature sequence, but reads corresponding to the mir-356 precursor sequence were found in the stem regions of its hairpin duplex, which are likely to be the true mature and star miRNAs (Supplemental Table S3). Other possibilities are that expression of these undetectable miRNAs is induced only for a short time in the life cycle, such as during the 1-cell-stage embryo (Stoeckius et al. 2009) or might be induced only under specific conditions or that they are hard to clone by the adapter-ligation-mediated method used in library preparation for Solexa because of terminal modifications on RNA molecules (Pak and Fire 2007).

In a previous study, we examined miRNA expression changes during larval development and in different sexes in C. elegans and found that the expression of many mature miRNAs is dynamically regulated during the developmental stages from embryo to young adult (Kato et al. 2009a). Therefore, we tested the overall changes in expression of mature miRNAs over time during aging. Pairwise comparisons between samples from aged populations (e.g., Day 5 vs. Day 8, or Day 5 vs. Day 12) showed strong correlations in their expression changes, while pairwise comparisons between samples from Day 0 young adult and any other aged populations exhibited large differences in their expression changes (Supplemental Fig. S1). These observations suggest that alterations in expression of miRNAs mostly occur in the early stages of adulthood rather than in middle to later stages of lifespan and do not fluctuate greatly from mid-adulthood.

For this reason, coupled with the availability of replicates we made in the deep-sequencing experiments (see Supplemental Fig. S2A,B for details), we focused on two stages, Day 0 young adults and Day 8 old adults in order to rank changes in expression of mature miRNAs during aging. We identified known miRNAs with statistically significant expression changes during aging $(P \leq 0.05)$ (Supplemental Table S2). These include $\sim 50$ mature miRNAs in total, which are equivalent to $\sim 30 \%$ of the 174 known, annotated mature miRNAs (miRBase release 14). Those with more than twofold changes in the number of sequence reads from Day 0 to Day 8 were listed in Figure 2A, which includes 23 up-regulated mature miRNAs and 16 downregulated ones, plus a mature miRNA variant for miR-71 (see the legend for Supplemental Table S2) and two miRNA star species (see below). As shown in Figure 2A, the most dramatically up-regulated miRNA was miR-239a, confirming our earlier report (de Lencastre et al. 2010). Another one of the miRNAs with the most dramatic up-regulation during aging was miR-34, which is highly conserved from 
A

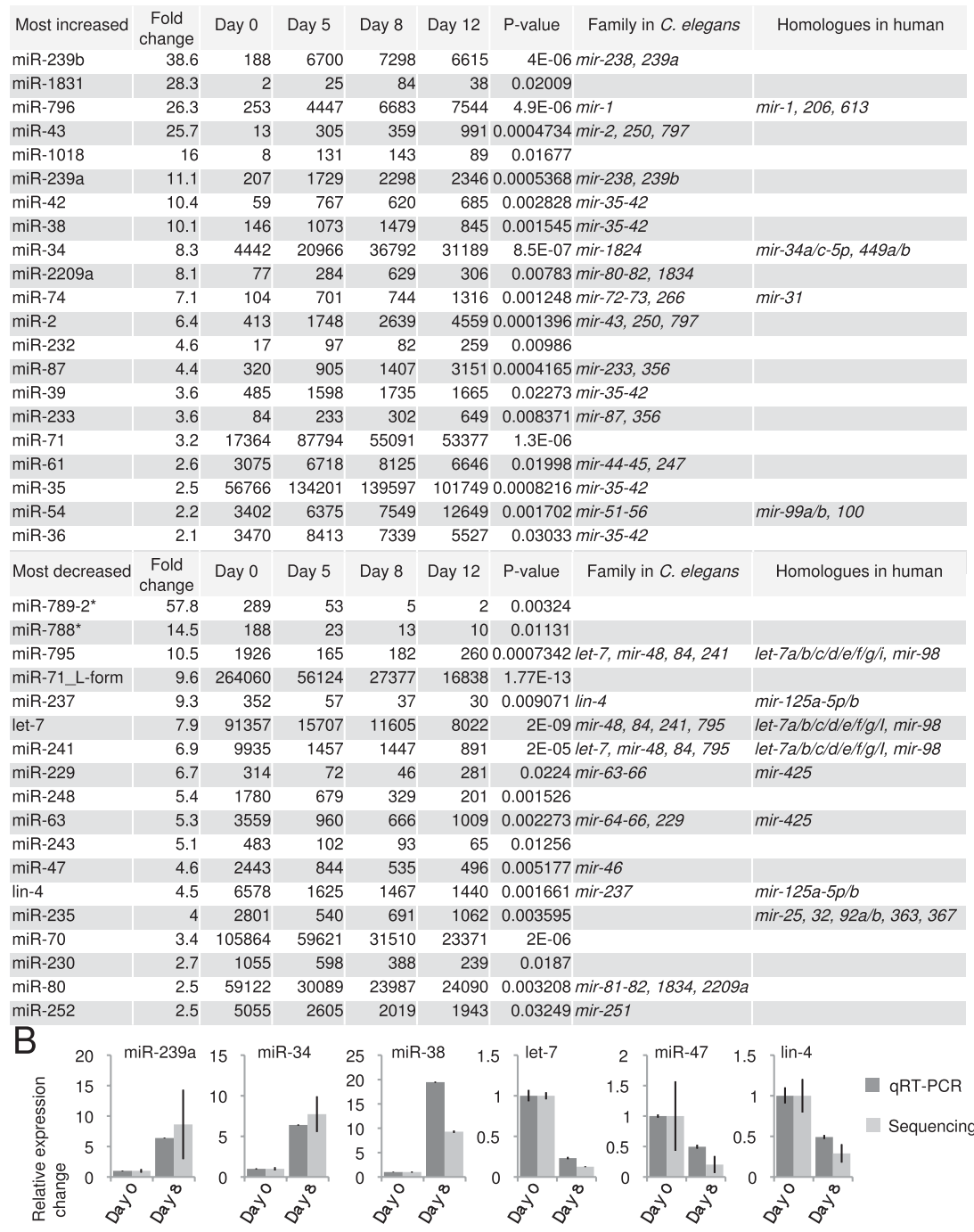

FIGURE 2. miRNAs with most increased and decreased expression during aging. $(A)$ Of the mature miRNAs with statistically significant expression changes during aging $(P \leq 0.05)$, those with more than twofold changes in the number of reads from Day 0 to Day 8 are shown here. This list also includes a mature miRNA variant for miR-71 and two miRNA star strands. miRNAs were sorted by the fold-changes. $P$-values are minimum $P$-value of pairwise analyses among Day 0, Day 5, Day 8, and Day 12 (see Materials and Methods for more details). Two of the miRNAs with ${ }^{\star}$ in their names represent miRNA star species, and sequences with the most abundant number of reads (Supplemental Table S3) were used for counting as we have done for the mature miRNAs. Note that the expression of annotated mature miR-71 (19 nt) appeared to be increased during aging as observed in the qRT-PCR experiments (Supplemental Fig. S2C). However, its longer form (miR-71_L-form) (23 nt) was more abundant than the annotated one, and it showed decreased expression during aging (Supplemental Table S3; details are discussed in the legend for Supplemental Table S2). (B) The results of miRNA expression changes were confirmed by qRT-PCR. The results were normalized by the average of the expression of act-3 and ama-1. Error bars for qRT-PCR results shown by dark gray bars indicate standard deviation (SD). Additional results are shown in Supplemental Figure S2C. Error bars for deep-sequencing results shown by light gray bars represent the maximum and minimum values in fold-changes in two replicates, which were calculated after each read was normalized by the total number of aligned reads in each library.

C. elegans to human and involved in the DNA damage response across phyla (He et al. 2007; Kato et al. 2009b). An example of the most down-regulated miRNA, lin-4, the first miRNA found to be necessary for normal lifespan in C. elegans (Boehm and Slack 2005), indeed, showed a significant reduction during aging. Additionally, another founding member of miRNAs, let-7, and its family miRNAs such as mir-241 and mir-795 (Roush and Slack 2008), showed a dramatic decrease in expression during aging (Fig. 2A).

We confirmed these results by biological replicates of the deep-sequencing libraries for Day 0 and Day 8, using independently prepared RNA samples, and also by quantitative RT-PCR (qRT-PCR) (Fig. 2B; Supplemental Fig. S2), supporting the reliability of our data. We further performed a simple hierarchical clustering analysis and found that miRNA family members have a similar trend in expression changes during aging (Supplemental Fig. S3). All together, these observations suggest that, in addition to lin-4, additional miRNAs might have important roles in lifespan regulation in C. elegans.

\section{Adult-specific loss of alg-1 activity disrupts miRNA expression changes and results in a shorter lifespan}

To test the importance of miRNAs in aging, we examined how the loss of activity of ALG-1 affects normal lifespan. The alg-1 gene encodes an Argonaute protein which is necessary for miRNA maturation and function but is not required for other known small RNA pathways (Grishok et al. 2001; Batista et al. 2008). In this experiment, spe-9(hc88) animals were first cultured on standard Escherichia coli OP50 bacteria and then exposed to feeding RNAi against alg-1 and empty vector L4440 as a control when they were Day 0 young adults (Supplemental Fig. S4). This enabled us to avoid the possibility that the effect of alg- 1 loss on the lifespan was due to a defect in development. We found that animals exposed to alg-1 RNAi displayed a significantly shorter lifespan compared to the control (Fig. 3A; Supplemental Table S4), demonstrating that miRNAs are necessary for normal lifespan in C. elegans adults. Essentially the same results were obtained when wild-type N2 animals were cultured with alg-1(RNAi) versus control at 
A
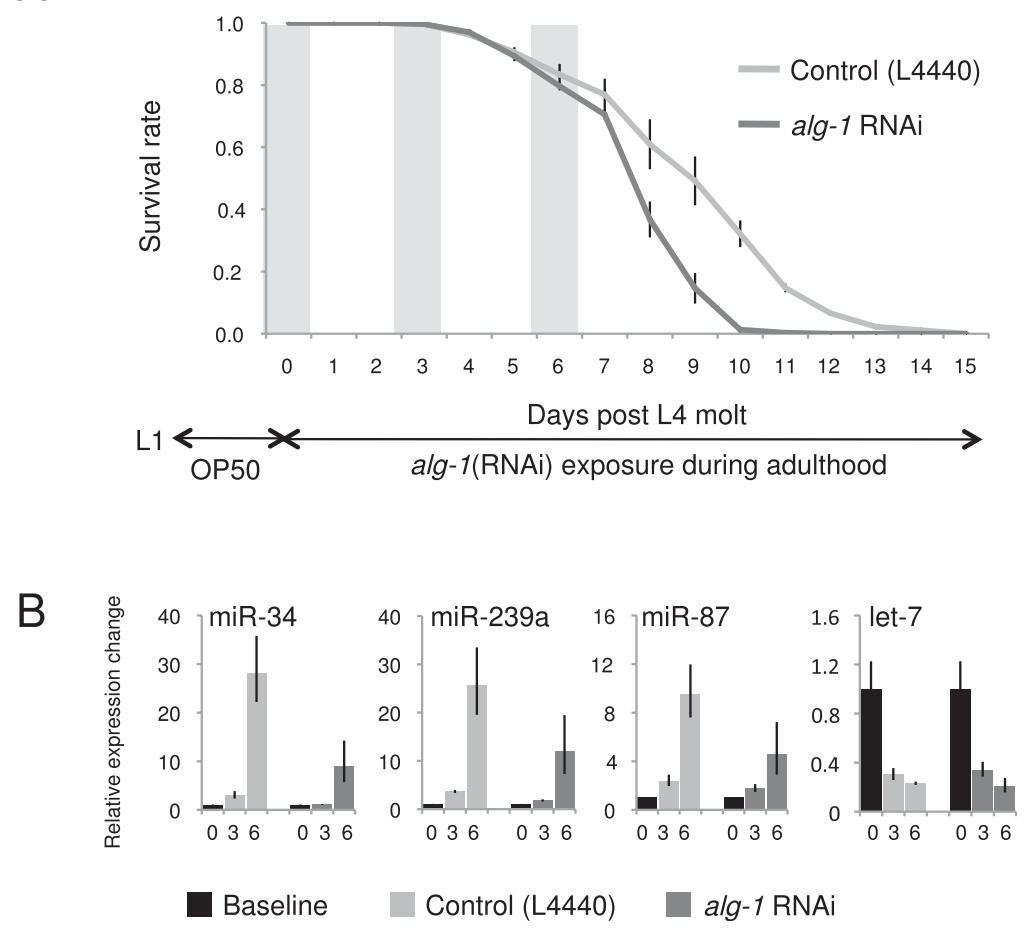

FIGURE 3. Lifespan of spe-9(hc88) animals treated with adult-specific RNAi against alg-1. (A) Animals were exposed to RNAi only during adulthood. Mean adult lifespans for the control and alg- 1 RNAi were $9.14+/-0.33$ and $7.89+/-0.15 \mathrm{~d}$, respectively $(P \leq 0.0005)$. Detailed results of the lifespan assay are shown in Supplemental Table S4. Error bars represent standard error (SE) calculated from triplicates. (B) Expression changes of age-associated miRNAs were examined by qRT-PCR. Data were normalized by the average of the expression levels of act-3 and ama-1 genes. Error bars represent SD. Day 0 animals cultured on OP50 bacteria (just before RNAi exposure) were used as a control.

either $20^{\circ} \mathrm{C}$ or $23^{\circ} \mathrm{C}$ (data not shown). This is further supported by the observations that miRNAs with increasing expression during aging were disrupted in their expression by loss of alg-1 activity, while those with decreasing expression, such as let-7, were not affected (Fig. 3B). Consistent with this conclusion, we recently found that some of the age-associated miRNAs identified here, indeed, play important roles in lifespan regulation in C. elegans (de Lencastre et al. 2010).

\section{Conditions that modify normal lifespan can modulate age-associated miRNA expression changes}

To further understand the contribution of these age-associated miRNAs to aging, we examined their expression changes under conditions that modified lifespan. Specifically, we tested whether conditions leading to a long lifespan could cause a delay in miRNA expression changes and if conditions leading to a short lifespan could accelerate their expression during aging. In C. elegans, an increase in temperature accelerates the aging processes and reduces lifespan, whereas a decrease in temperature causes a delay in the aging processes and prolongs lifespan (Klass 1977).
In this study, synchronized spe-9(hc88) animals were first cultured at the nonpermissive temperature $\left(23^{\circ} \mathrm{C}\right)$ during larval development to induce their sterility and then shifted to $15^{\circ} \mathrm{C}$ and $27^{\circ} \mathrm{C}$ when they were Day 0 young adults. Compared to control animals which were kept at $23^{\circ} \mathrm{C}$ during all life stages including adulthood, animals shifted to the lower temperature $\left(15^{\circ} \mathrm{C}\right)$, indeed, showed a significantly longer lifespan, while those exposed to the higher temperature $\left(27^{\circ} \mathrm{C}\right)$ resulted in a shorter lifespan (Fig. 4A). Where possible, we purified total RNAs from Day 0, Day 3, Day 5, Day 8, Day 12, and Day 15 animals at each temperature condition and examined changes in expression of ageassociated miRNAs using qRT-PCR.

We found that miRNAs with increasing expression during aging showed a significant delay in expression change at the long-lived $15^{\circ} \mathrm{C}$ condition, while their expression changes were accelerated at the short-lived $27^{\circ} \mathrm{C}$ condition (Fig. 4B, left; Supplemental Fig. S5). For example, miR-34 and miR-239a, both of which exhibited about a 10 - to 15 -fold increase in expression from Day 0 to Day 8 at the standard condition $23^{\circ} \mathrm{C}$, reached a similar fold increase at Day 15 in the long-lived condition, while they reached a similar fold increase earlier at Day 3 in the short-lived condition (Fig. 4B, left). These observations demonstrate the tight regulation of miRNA expression during aging and support the idea that maintaining these miRNAs at lower levels during aging might contribute to a delay in the aging process and cause longer lifespan or health span. Also, these results suggest their potential as molecular biomarkers for aging, although we cannot rule out that some of them may have been affected by higher temperature rather than short lifespan, including the case of lin-4 in Figure 4B, right.

In contrast to miRNAs with an increase in expression during aging, those with a decrease in expression during aging did not show such a delay; they exhibited the same rapid decrease in expression in the long-lived condition at $15^{\circ} \mathrm{C}$ as observed in the standard control condition (Fig. 4B, right). All miRNAs examined, including let-7 and lin-4, reduced their expression a short time after animals were shifted to the lower temperature (Fig. 4B, right; Supplemental Fig. S5). One possible explanation is that their down-regulation might be programmed at earlier stages including during larval development. Alternatively, their expression might be controlled independently from the length of lifespan. 
A

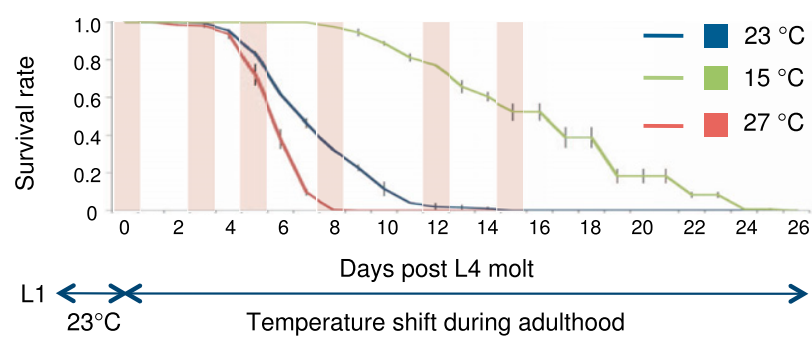

B
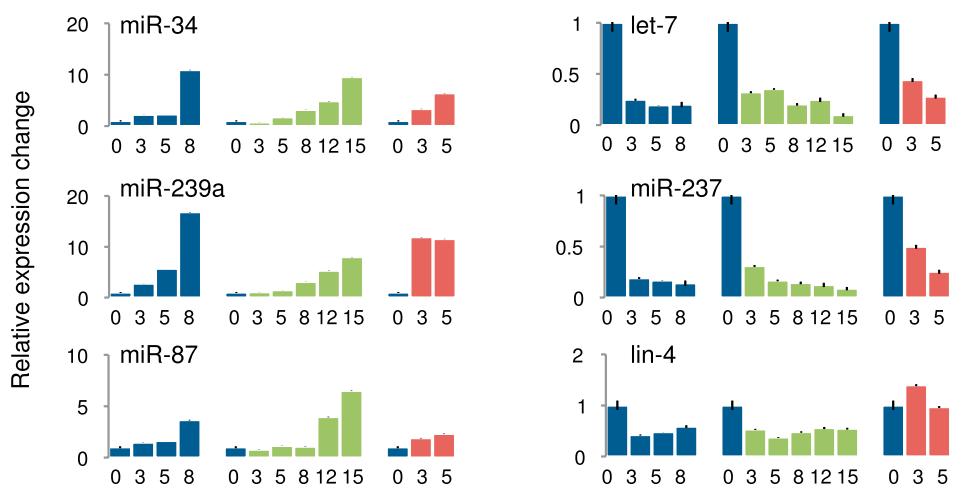

2 lin-4

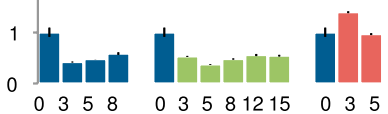

C
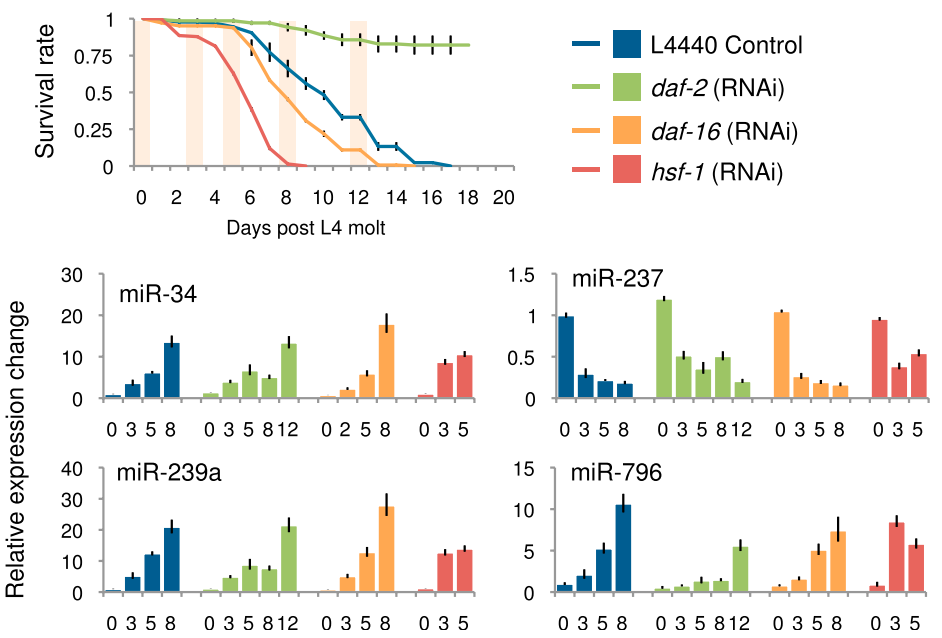

FIGURE 4. Expression changes of age-associated miRNAs in conditions that modify lifespan. (A) Lifespan of spe-9(hc88) animals was assayed at different temperatures, $15^{\circ} \mathrm{C}, 23^{\circ} \mathrm{C}$, and $27^{\circ} \mathrm{C}$. Mean adult lifespans were $7.62+/-0.15 \mathrm{~d}, 16.21+/-0.37 \mathrm{~d}$, and $6.11+/-0.17 \mathrm{~d}$ at $23^{\circ} \mathrm{C}, 15^{\circ} \mathrm{C}$, and $27^{\circ} \mathrm{C}$, respectively $(P<0.0001)$. Error bars indicate SE. Details are shown in Supplemental Table S4. Total RNAs were purified from Day 0, Day 3, Day 5, Day 8, Day 12, and Day 15 post-L4 molt as highlighted. (B) Expression changes of age-associated miRNAs were examined by qRT-PCR. Data were normalized by the average of the expression levels of act-3 and ama-1 genes. Error bars represent SD. Day 0 animals cultured at $23^{\circ} \mathrm{C}$ (just before the temperature shift) were used as a control. Additional results are shown in Supplemental Figure S5. (C) Similar to the temperature-shift experiment, a delay or acceleration in expression changes of the age-associated miRNAs was observed in genetically modified lifespan backgrounds. In this study, spe-9(hc88) animals were exposed to each feeding RNAi, including the control empty vector (L4440), from the L1 stage, in order to induce a sufficient RNAi effect.

Moreover, we confirmed the tight regulation of ageassociated miRNAs in modified lifespan backgrounds induced by RNAi-mediated knockdown of aging-related genes. These include $d a f-2$, and $d a f-16$ and $h s f-1$, which cause long- lived and short-lived conditions, respectively, when their activity is diminished (Fig. 4C; for review, see Antebi 2007; Kenyon 2010). Similar to those observed in the temperature-shift experiments above, many age-associated miRNAs with up-regulated expression during aging, such as miR-239a, exhibited a delay in expression changes, while those with down-regulation during aging showed expression changes independently of their lifespan conditions (Fig. 4C), although we found that some of the age-associated miRNAs were not consistent with such trends in expression changes, possibly due to their genetic interaction with the aging-related genes examined (M Kato and FJ Slack, unpubl.).

\section{Age-associated miRNA expression is mainly attributed to transcriptional activity from miRNA promoters}

We validated the altered expression of age-associated miRNAs using transgenic animals carrying constructs of the miRNA promoter fused to a GFP marker gene. Transgenic lines used in this study contained transgenes integrated into chromosomes and were crossed into the spe9 (hc88) mutant background. We tested changes in GFP signal intensity during aging at Day 0, Day 3, and Day 6 of adulthood. Most of the lines we examined exhibited similar trends in expression change shown for the mature miRNAs from the results of deep-sequencing and qRT-PCR. For example, mir-34, which showed a dramatic increase in expression of the mature miRNA during aging, exhibited a similar increase in GFP signals in aged transgenic animals (Fig. 5). For let-7, which showed a marked reduction of mature miRNA levels during aging, we found that GFP signals were nearly absent in Day 6 animals (Fig. 5). This suggests that at least a part of these expression changes can be attributed to transcriptional activity driven by the miRNA promoter.

Interestingly, in many cases of miRNAs up-regulated with age, the GFP signals seem to be expressed in tissues or cells in which they are not expressed at earlier stages. The GFP signals from the mir-34::gfp transgenic line were detectable de novo in cells on the ventral side in most 

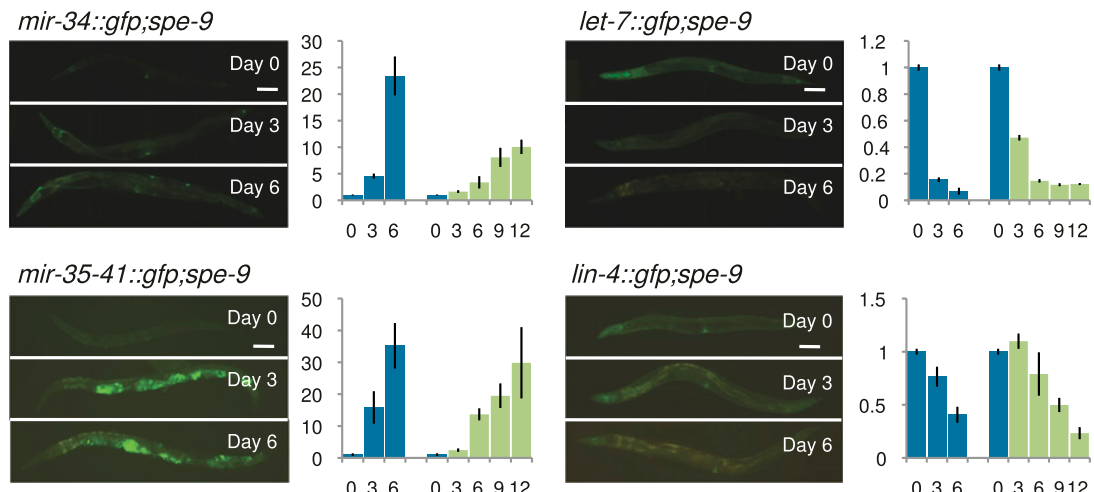

lin-4::gfp,spe-9
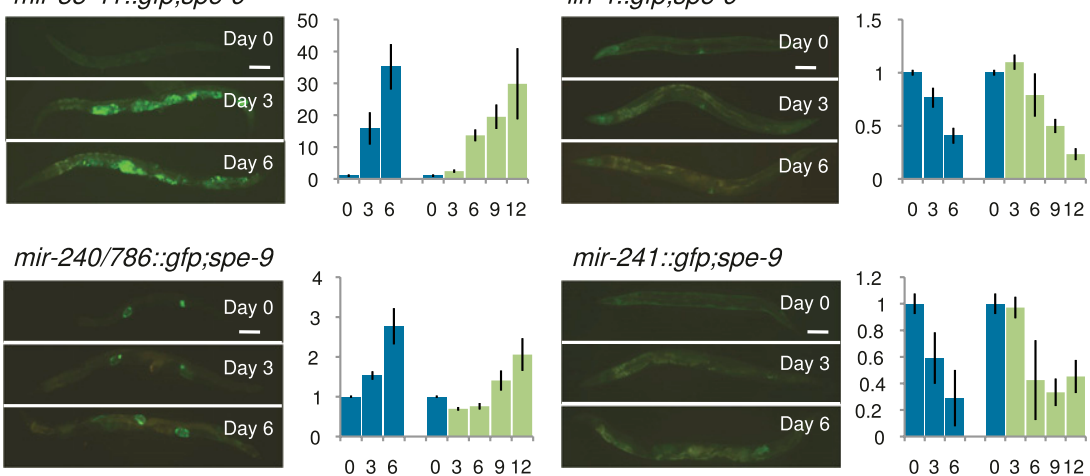

mir-241::gfp;spe-9
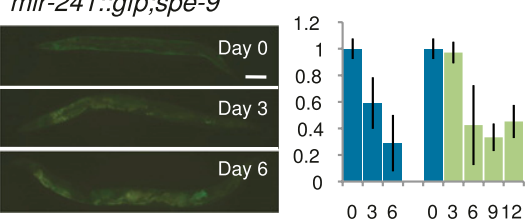

FIGURE 5. Age-associated changes in GFP signals expressed from miRNA promoter::gfp transgenes. The vertical axis represents a fold-change in GFP signal intensity, microscopically determined from $\sim 15-20$ individual whole animals at each time point for each line. Error bars represent SE. The blue-colored and green-colored bars represent the GFP signal intensity observed in the transgenic lines cultured at the standard temperature $23^{\circ} \mathrm{C}$ and the long-lived condition at $15^{\circ} \mathrm{C}$, respectively. Scale bars represent $100 \mu \mathrm{m}$. Additional results for miRNA::gfp lines were examined, and strain information is shown in Supplemental Figure S6B.

aging animals (Fig. 5; marked by arrowheads in larger images in Supplemental Fig. S6B). In another example, the mir-35-41 miRNA cluster, which is highly expressed in developing embryos (Lim et al. 2003), exhibited extensive GFP signals in mid- to late- aging animals, in spite of their lack of fertilized embryos in the spe-9(hc88) background. These changes in expression pattern could be due to the following possibilities: a tightly regulated aging activity; transgene-specific background expression of GFP signals; the effect of disorganized tissues with age (e.g., diffusion of GFP proteins); or uncontrolled transcription because of an age-dependent loss of proper regulation of gene expression (Lund et al. 2002). If the GFP signals specifically observed in aged animals are due to age-related uncontrolled transcriptional activation, this erratic activation of miRNA expression might trigger unfavorable age-related decline, since miRNAs affect activities of many target genes.

We also examined age-related changes in the promoter::GFP signal intensity in the lower temperature-induced longer lifespan background, as we did for mature miRNAs. As expected, the GFP signals expressed from miRNA promoter::gfp lines for up-regulated miRNAs in aging (e.g., mir-34) exhibited a remarkable delay in their changes in the long-lived condition at $15^{\circ} \mathrm{C}$, compared to those observed in the control cultured at the standard condition $23^{\circ} \mathrm{C}$ (Fig. 5; images are shown in Supplemental Fig. S6C). In addition, the transgenic lines for down-regulated miRNAs in aging (e.g., let-7) also showed a delay in changes of the GFP signals in the long-lived condition, unlike the results ob- served for the mature miRNAs (Figs. 4 and 5; images are shown in Supplemental Fig. S6C). However, the extent of delay in changes of the GFP signals detected for down-regulated miRNAs was not as pronounced, compared to those detected for up-regulated miRNAs, and may reflect the stability of the GFP protein.

\section{Computational miRNA target prediction combined with transcriptome analysis reveals likely candidate genes regulated by age-associated miRNAs}

We predicted candidate targets of ageassociated miRNAs and their possible biological pathways. miRNAs negatively regulate their target genes by binding to complementary sequences in the $3^{\prime}$ UTR of mRNAs (Vella et al. 2004). Although miRNAs had been thought to preferentially affect the level of proteins rather than mRNAs, it has been shown that miRNAs also reduce the level of mRNAs (Bagga et al. 2005; Guo et al. 2010). Indeed, microarray experiments have proven to be an effective way to find genes modulated by miRNAs (Johnson et al. 2007). We assumed that age-associated alterations of miRNA expression levels might cause a reciprocal trend in the expression change of the mRNAs of their target genes during aging. Thus, in addition to the conventional target prediction algorithms such as miRanda, we combined the expression profiles of miRNAs and protein-coding genes in aging and age-related phenotypic information together (details are described in Materials and Methods) (Fig. 6A) to highlight candidate target genes with reciprocal expression changes to their cognate miRNAs during aging. Three hundred and fifty-four protein-coding genes were predicted as targets for one or multiple age-associated miRNAs (from Fig. 2A) (with a maximum of 24 different miRNAs found on one target) (Supplemental Table S5). Of these 354, 58 genes are known to be necessary for normal lifespan in C. elegans, suggesting that these 58 genes are better candidates for regulation by age-associated miRNAs (these are highlighted in red in Supplemental Table S5). Next, to determine how these predicted targets contribute to aging, we performed pathway enrichment analysis with KEGG (Kyoto Encyclopedia of Genes and Genomes). KEGG is an integrated database resource providing functional aspects of biological systems, such as biological pathways in cells and organisms, based on the molecular information of genes and their products (Kanehisa et al. 2010). For C. elegans, $\sim 1700$ genes are classified into 121 pathways in the KEGG database. In our 
A
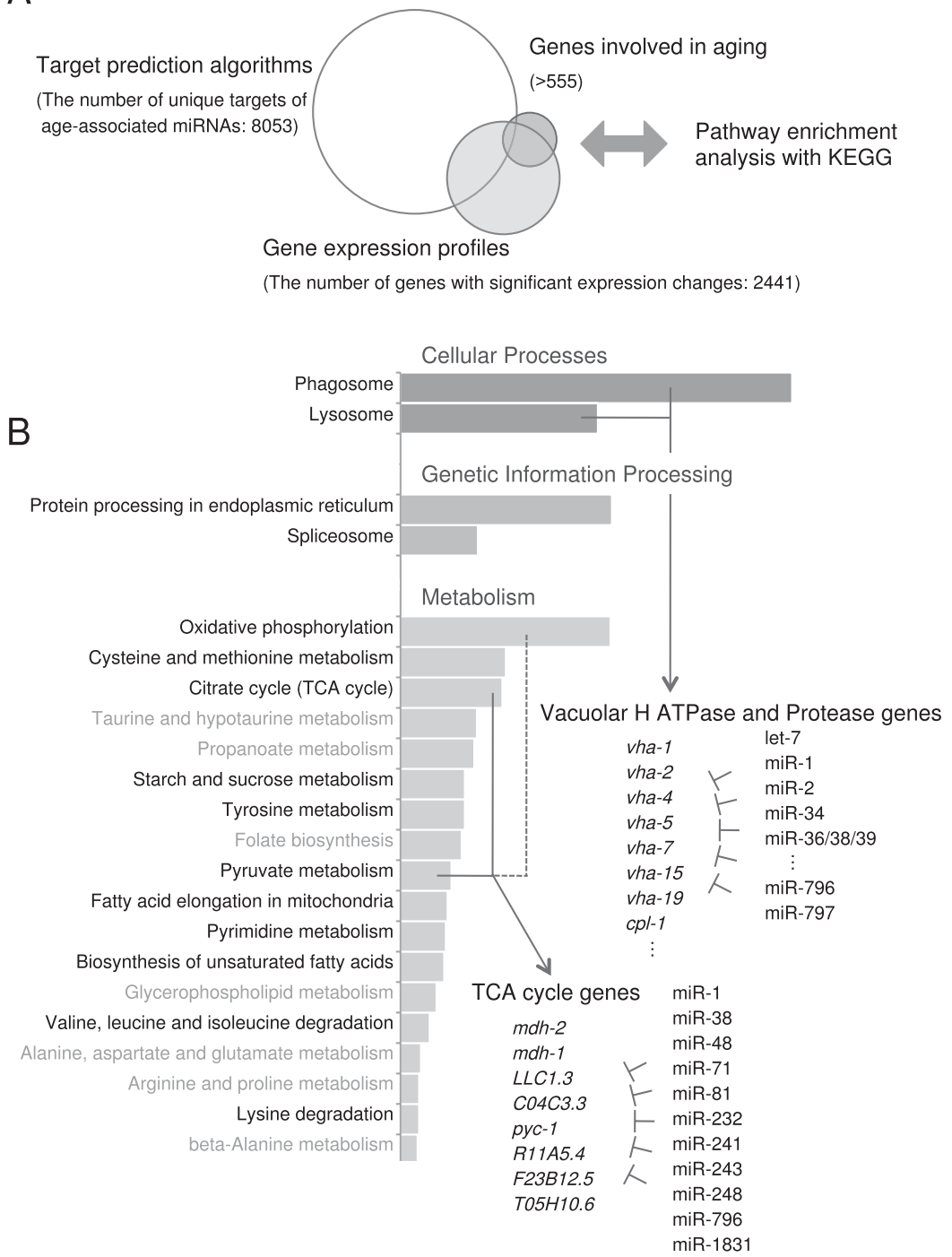

FIGURE 6. Target prediction of age-associated miRNAs and pathway analysis. (A) Target prediction algorithms combined with gene expression profiles and age-related phenotypes followed by the pathway analysis revealed strong candidate targets of age-associated miRNAs and their possible roles in aging. The expression profiles for protein-coding genes were obtained from Budovskaya et al. (2008), where they used a spe-9(hc88) background and similar conditions for RNA preparation, including the time points during aging. The size of each circle reflects the number of genes identified in each category (but the area of overlapped regions is not to scale completely). (B) Pathway enrichment analysis with KEGG against predicted target genes identified their possible roles in aging processes. The results were sorted by the order of enrichment (number of predicted genes in each pathway). For example, many TCA cycle and related genes were predicted as targets. These serve an important function in mitochondria together with genes classified in oxidative phosphorylation (shown by a broken line). The names of gene pathways shown in black represent genes necessary for the normal lifespan, suggesting that these pathways are involved in aging. Detailed results are available in Supplemental Tables S5 and S6.

pathway analysis, predicted target genes of 33 age-associated miRNAs were found to be significantly enriched in 22 pathways $(P<0.05)$ (Fig. 6B; Supplemental Tables S5, S6). Importantly, these include phagosome and lysosome pathways, which contain vacuolar family and proteasome genes, suggesting that age-associated miRNAs including let-7 and mir-1 may contribute to aging through the control of the proteasome system. Also, we found that genes in the tricarboxylic acid (TCA) cycle and oxidative phosphorylation, which form the core for energy production in the mitochondria (Wallace 2005), are predicted as targets of age-associated miRNAs (Fig. 6B). Indeed, mitochondrial function and proteostasis are coordinately modulated during aging by insulin, target of rapamycin (TOR), and sirtuin signaling pathways to protect genomes and cells from reactive oxygen species and misfolded proteins (Haigis and Yankner 2010).

\section{miRNA "star" strands preferentially accumulate in RDE-1, and they change their expression during aging}

As we mentioned earlier, $\sim 70 \%$ of aligned sequence reads mapped to known, annotated mature miRNAs, and $\sim 15 \%$ mapped to unannotated genomic regions, suggesting that there still might be age-associated sncRNAs in this fraction. This fraction could include novel miRNA candidates and miRNArelated sequences such as miRNA "star" molecules.

The miRNA star sequence is the opposite strand to the mature one in a hairpin duplex derived from the miRNA precursor, and it has been previously thought that they are byproducts in miRNA processing and degraded rapidly. However, several recent studies demonstrate that they are incorporated into Argonaute protein complexes in Drosophila (Czech et al. 2009; Okamura et al. 2009; Ghildiyal et al. 2010). Indeed, our deep-sequencing libraries detected a significant number of reads corresponding to miRNA hairpin-loop sequences, including many miRNA star reads. Star reads and their derivatives were detected in our library for 111 known miRNAs (miRBase release 14), and their levels generally decreased during aging, similar to those of mature miRNAs (Supplemental Table S3). These observations suggest that star strands can, indeed, exist as stable RNA molecules in C. elegans.

To investigate in which Argonaute proteins the star miRNA strands are incorporated, we examined the enrichment of sequence reads corresponding to mature and star 
miRNAs in ChIP-Seq (chromatin immunoprecipitation followed by deepsequencing) libraries made for Argonaute proteins, ALG-1, ALG-2, and RDE-1 (Corrêa et al. 2010) (sequence data were obtained from the Genome Expression Omnibus [GEO], accession: GSE20649). In addition to ALG-1, another Argonaute protein, ALG-2, is thought to act redundantly with ALG-1 to support it in miRNA processing (Tops et al. 2006). A third member, RDE-1, is involved in the double strand RNA-induced siRNA pathway (Tabara et al. 1999). Consistent with the previous report (Corrêa et al. 2010), most of the reads bound to these Argonaute proteins were found to be miRNAs. Notably, we found that the miRNA reads corresponding to star strands were significantly enriched in the RDE-1 ChIP-Seq library compared to those in ALG-1 or ALG-2 (Supplemental Fig. S7A; Supplemental Table S7) and also compared to the ratio of mature and star miRNAs in our deep-sequencing results from aging samples (mature: 99.85\%, and star: 0.15\%) (Supplemental Table S3). Additionally, the accumulation of star miRNAs as well as mature ones was reduced in $r d e-1$ mutants compared to wild-type animals (Supplemental Fig. S7B; Supplemental Table S7) (sequence data were obtained from GEO, accession: GSE20649) (Corrêa et al. 2010). A similar reduction of miRNA star level in $r d e-1$ mutants was also observed in another library obtained from the GEO, GSE19414 (Gent et al. 2010) (data not shown). These results suggest that the star miRNA strands are preferentially sorted into or stabilized in RDE-1 Argonaute protein in C. elegans.

As observed in our deep-sequencing libraries, in most cases, the star strands accumulated at much lower levels than their corresponding mature sequences. However, we found some cases where both species appeared to accumulate at a similar frequency and others where star reads were much more abundant than the reads for the mature miRNA (Fig. 7A; Supplemental Table S3). For each miRNA with statistically significant expression changes during aging in either mature or star strand, we tested the correlation between mature and star miRNA strands over the time course of aging (Fig. 7B). Of those, seven significantly changed the expression of their star strands (red- or green-colored dots in Fig. 7B). These include miR-788 and miR-789-2, both of which have more abundant star strands than mature ones and have a similar trend in expression change (Figs. 2A and 7B,C, top). Importantly, there are several cases where the star strands showed distinct changes in abundance relative to their mature counterparts during aging, including those for miR-34 and miR63 (Fig. 7B,C, bottom; Supplemental Table S3), suggesting the complexity in miRNA processing. Recently, it has been shown that star miRNA strands also have a capacity to repress synthetic targets in vitro (Yang et al. 2010). Although the biological significance of star strands is still not clear, our findings suggest that they might serve as regulatory molecules in aging in C. elegans, similar to mature miRNAs. 


\section{Novel miRNA candidates were identified from aging animals}

In the process of filtering out annotated sequence reads, we were left with $\sim 30,000$ unique, unannotated sequencing reads. These potentially include novel miRNA candidates and additional age-associated sncRNAs. Computational prediction with the miRDeep program (Friedlander et al. 2008) yielded 76 novel miRNA candidates from these unannotated reads (Supplemental Table S8). For 21 of these, we detected corresponding star strands or their derivative reads in our data set. Based on the features, including the presence of star strands or expression change during aging (Supplemental Tables S8S10), we picked seven novel miRNA candidates for further verification (Fig. 8A). As shown in Figure 8B, some of the candidates may fall into known miRNA families because they had the same "seed" sequences as other known miRNAs. Also, qRT-PCR experiments showed that some of them change their expression during aging (Fig. 8C). Additionally, we constructed candidate miRNA promoter:: $g f p$ fusion transgenic lines and detected GFP signals in adult C. elegans tissues, such as sensory neurons in the head (Supplemental Fig. S6D), demonstrating that these sequences are transcribed.

In order to further validate whether these candidates are bona fide miRNAs, we examined their expression level in mutants of alg-1(gk214), which is necessary for miRNA maturation (Grishok et al. 2001). We found that five of the candidate miRNAs examined significantly reduced their accumulation in the alg-1 (gk214) mutant background, indicating that they are real miRNAs (Fig. $8 \mathrm{D}$, top). However, for two candidates, 405191_adh and 1263561_spe, we could not validate these as real miRNAs since they did not show reduced expression in the alg-1 (gk214) or alg-2(ok304) mutants (Fig. 8D). Although we attempted to test their expression in an alg-2(ok304); alg-1(RNAi) background, this genetic combination led to growth arrest at an early larval stage. Eventually, nine novel miRNA candidates were officially annotated as miRNAs by miRBase, which include mir-5545 to mir-5553 (Fig. 8; Supplemental Tables S8-S10).

A

B

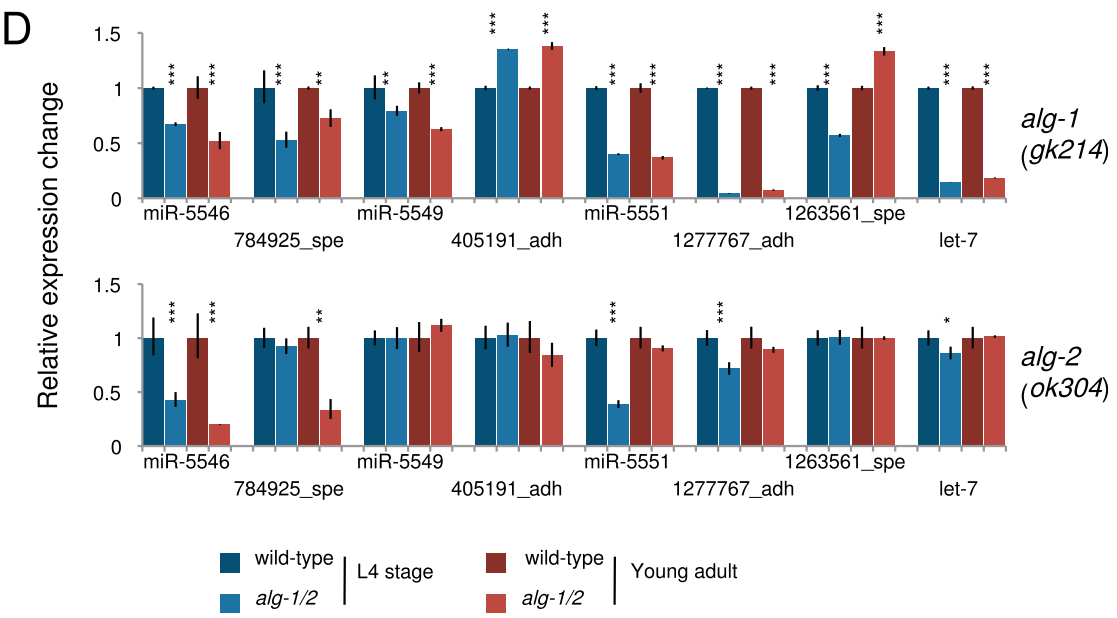

FIGURE 8. Characterization of novel miRNA candidates. (A) The secondary structures of primary miRNA precursors were predicted for novel miRNA candidates using the RNAfold program. Note that mir-5546 (1128878_adh) was also reported in Stoeckius et al. (2009), although it has not yet been submitted to miRBase or WormBase. (B) Some novel miRNA candidates may fall into known miRNA families since they have the same "seed" sequence as known miRNAs. $(C)$ The age-associated expression changes of novel miRNA candidates were confirmed by qRT-PCR. The results were normalized by the average of the expression of act-3 and ama-1. Error bars indicate SD. $(D)$ Levels of novel miRNA candidates were examined by qRT-PCR in mutants of Argonaute family genes [top: alg-1(gk214) and bottom: alg-2(ok304)] at two different developmental stages - the fourth larval (L4) and young adult. The results were normalized by the expression level of U18 and standardized to the level in wild-type N2 in each stage examined. Error bars indicate SD. $P$-values were calculated by $t$-test $\left({ }^{\star * *}: P<0.0001\right.$; ${ }^{* *}$ : $\left.P<0.001 ;{ }^{*}: P<0.05\right)$.

\section{Additional sncRNAs are associated with aging}

We hypothesized that our deep-sequencing approach would reveal additional sncRNAs related to the aging process. Of the $\sim 30,000$ unique, unannotated reads, the 14,500 unique 
reads 20 nucleotides (nt) or longer in size were grouped based on features, such as expression change, chromosomal location, and nucleotide content (Supplemental Fig. 8A,B; Supplemental Table S11). We found that $\sim 100$ of them significantly changed their expression during aging. Notably, many of these age-associated reads started with a uracil (U) or a guanine $(\mathrm{G})$ and include sequence reads of $21 \mathrm{nt}$ starting with a $\mathrm{U}$ (referred to here as $21 \mathrm{nt}-\mathrm{U}-\mathrm{RNA}$ ) and sequence reads of $22 \mathrm{nt}$ and $26 \mathrm{nt}$ starting with a $\mathrm{G}$ (referred to here as 22nt-G-RNA and 26nt-G-RNA, respectively) (Fig. 9A).

21nt-U-RNAs contain a subset that includes 21U-RNAs/ piRNAs, a class of sncRNAs involved in transposon silencing in the germline of flies, C. elegans, and mammals (Batista et al. 2008; Stefani and Slack 2008). Since typical 21URNAs in C. elegans are preferentially localized on chromosome IV and have the core consensus motif "CTGTTTCA" in their upstream regions, we searched for these features

A

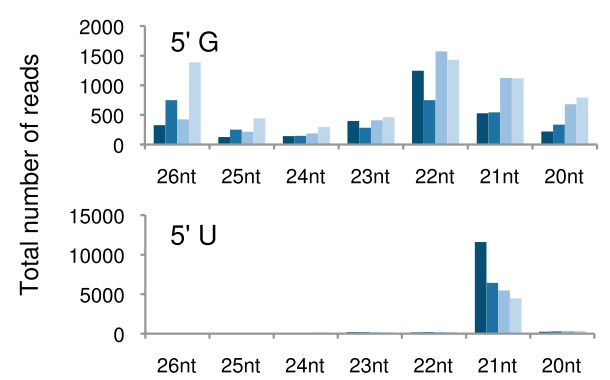

Day 0 Day $5 \quad$ Day $8 \quad$ Day 12

C

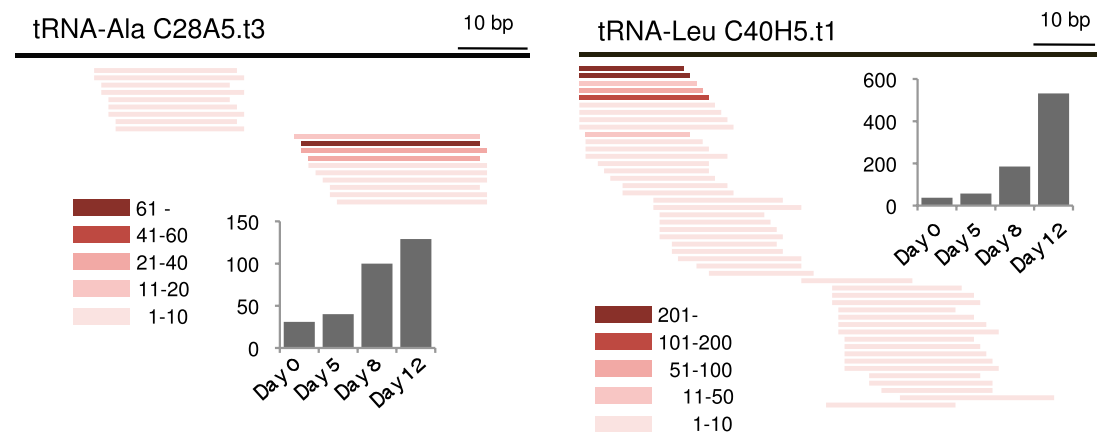

FIGURE 9. Characterization of additional sncRNA candidates. (A) The total number of unannotated reads starting with a $U$ or a $G$ was examined in each aging stage. 21nt-U-RNAs showed a decrease in expression with age, while 26nt-G-RNAs and their related reads appear to be increased. $(B)$ The total number of $21 \mathrm{U}-\mathrm{RNA} / \mathrm{piRNA}$ reads, including both known and novel ones, was reduced during aging. $(C)$ tRNA-derived fragments were increased in their accumulation during aging, and two examples are shown here. The longest bars represent the annotated mature tRNA sequences, and each shorter bar represents unique sequence reads corresponding to a part of their mature products. The scores and the color gradation on each shorter bar indicate the number of sequencing reads for each read found in all aging samples. The bar graphs represent the expression changes of the total number of tRNA-derived fragments during aging. All examples are shown in Supplemental Table S15. and identified an additional nine novel 21U-RNA candidates. These novel 21U-RNAs, as well as known, annotated ones, showed a gradual decrease in expression during aging (Fig. 9B; Supplemental Table S12). Furthermore, although our remaining $21 \mathrm{nt}-\mathrm{U}$-RNAs are not from chromosome IV and/or do not have the perfect canonical consensus motif, they still appear to be bona fide $21 \mathrm{U}$-RNAs since $\sim 300$ of them show reduced expression in a prg-1 mutant, which is cessary for 21U-RNAs/piRNAs biogenesis (sequence data prg-1 mutants were obtained from GEO, accession: (Batista et al. 2008; Supplemental Fig. S8C; Sup-

Unlike these 21nt-U-RNAs, which showed a decrease in expression during aging, both 26nt-G-RNAs and 22ntG-RNAs and their derivatives that we cloned appear to be increased during aging (Fig. 9A, top; Supplemental Table S11), although for many, their expression levels were quite low and fluctuated during aging. In total, we cloned 2082 22nt-G-RNAs and 525 26nt-G-RNAs in our libraries (Supplemental Table S14).

These possibly age-associated 26ntG-RNAs and 22nt-G-RNAs that we cloned may correspond to a class of recently identified endo-siRNAs, 22Gand 26G-RNAs, which are implicated in transposon silencing and chromosome segregation in C. elegans (Claycomb et al. 2009; Gu et al. 2009). To address this question, we investigated their accumulation in mutants of WAGO and CSR-1 pathways, such as $d r h-3$ and $c s r-1$, which are necessary for the generation of these endo-siRNAs. We utilized sequence data for these mutants obtained from GEO, accession: GSE18215 and GSE18165 (Claycomb et al. 2009; Gu et al. 2009). 22nt-G-RNAs showed a dramatic reduction in their accumulation in mutants including $d r h-3$, compared to the control wild-type animals (Supplemental Fig. S8D; Supplemental Table S14). Also, another abundant RNA species in aged animals, 26nt-G-RNAs, exhibited a marked reduction in their accumulation in mutants including $c s r-1$. These results indicate that the 22ntG-RNAs and 26nt-G-RNAs that we cloned from an aged animal population correspond to the recently identified endo-siRNA species (detailed observations are discussed in the legend for Supplemental Fig. S8D).

It is still not clear why these 26ntG-RNAs/26G-RNAs and 22nt-G-RNAs/ 
22G-RNAs seem to be increased during aging and how they are related to lifespan regulation, since spe-9(hc88) animals exposed to RNAi against genes involved in their biogenesis, such as csr-1 and $d r h-3$, did not show obvious lifespan abnormalities (data not shown). However, it has been shown that CDE-1 (also known as CID-1 or PUP-1), one of the factors necessary for the function of CSR-1 22G-RNAs, has a role in lifespan regulation and thermotolerance, together with DNA check point proteins, including CHK-1 and CDC-25.1 (Olsen et al. 2006), suggesting the possibility that these endo-siRNAs may also contribute to aging and stress response through the maintenance of genome stability and genome surveillance.

While the total levels of miRNAs and 21U-RNAs/ piRNAs showed a gradual decrease in accumulation during aging, other reads corresponding to known noncoding RNAs, such as tRNAs, rRNAs, and small snoRNAs, showed a gradual but constant increase in accumulation during aging (Fig. 1B; Supplemental Table S1). One would expect that these may be products of degradation since small RNAs ranging in size from 15-30 nt were selectively purified in the process of cDNA library preparation. However, at least in the case of some tRNAs and snoRNAs, they are unlikely to be simply derived from random degradation because some specific fragments preferentially accumulate, revealing that the cleavage events occur at specific sites in the mature tRNAs and snoRNAs (Fig. 9C; Supplemental Fig. S9; Supplemental Tables S15, S16). Also, as shown in the bar graphs in Figure 9C, for different types of tRNAs, their cleavage products all showed an increase in accumulation through aging.

It has been reported that tRNA fragments are present in a wide variety of organisms and often increase under various stress conditions such as heat and oxidative damage (Thompson and Parker 2009a). The nucleases responsible for tRNA cleavage during stress have been identified in budding yeast and mammalian cells, and both of these ribonucleases are found to cleave not only tRNAs but also rRNAs in response to stress (Thompson and Parker 2009b; Yamasaki et al. 2009). Most recently, it was demonstrated that stress-induced tRNA cleavage depends on a DNA methyltransferase Dnmt2, and Dnmt2-mediated methylation protects tRNAs against ribonuclease cleavage in Drosophila (Schaefer et al. 2010). The biological roles of these novel, cleaved RNAs remain largely unknown; however, their induction found in this study might be a consequence of accumulated damage with age.

\section{DISCUSSION}

Small, noncoding RNAs, including miRNAs, are key factors in the control of gene expression and in the maintenance of genome stability, and here we provide evidence that they contribute to the regulation of aging processes in C. elegans. Our expression profiling of sncRNAs using deep-sequenc- ing technology reveals new molecular features related to aging. We show in this study that many classes of small RNAs, including miRNAs, exhibited significant expression changes during aging in C. elegans. Also, we identified miRNA star strands, novel miRNAs, tRNA- and snoRNA-derived short fragments, and endo-siRNAs such as 21U-, 22G-, and 26G-related RNAs as differentially expressed during aging.

One of the most straightforward approaches to understanding the role of small RNAs in aging is to test the effects on lifespan of their knockout mutants. This is not generally a plausible approach for the thousands of $21 \mathrm{U}$-, 22G-, and 26G-RNAs but is plausible for the hundreds of miRNAs. In fact, in addition to the necessity of alg1-mediated miRNA processing in the normal lifespan, we recently found that some of the miRNAs with changes in expression during aging result in abnormal lifespan when they are deleted (de Lencastre et al. 2010). However, $\sim 40 \%$ of known miRNAs share significant sequence similarities (74 of 174 miRNAs [miRBase ver. 14.0] have the same "seed" sequences with other C. elegans miRNAs) (Supplemental Table S2), suggesting considerable potential for functional redundancy in miRNA families. Furthermore, a single gene may be regulated redundantly by different miRNAs at the same time, as genes often have multiple miRNA binding sites. This complexity makes it difficult to reveal biological roles of individual miRNAs using their genetic knockouts. However, analyses of miRNA expression and target prediction, followed by pathway analysis shown in this study, suggest that the miRNAs identified here function in known aging processes, such as proteostasis. Further, expression profiling of miRNAs in longevity mutants or on RNAi-mediated knockdown of aging-associated genes (e.g., daf-2 for long-lived and daf-16 for short-lived conditions) uncovered additional evidence for miRNA roles in aging.

The miRNA processing machinery yields both mature and star strands from the same hairpin-loop precursor products, and so one would expect that both mature and star miRNA strands have the same trend in their expression patterns. However, we found that some miRNAs have different trends in expression for their mature and star strands during aging. Additionally, we found that star miRNA strands preferentially accumulated in a third AGO protein, RDE-1, which is involved in the siRNA pathway. This feature might be highly conserved among diverse species since it was also recently reported in Drosophila that mature miRNA strands are predominantly sorted into AGO1, and their star strands accumulate in AGO2, which preferentially acts in the siRNA pathway, and this strand selection is associated with central base-pairing mismatches in miRNA precursor duplexes (Okamura et al. 2009). Also, the same group recently demonstrated that star strands have a capacity to repress expression of synthetic targets in vitro (Yang et al. 2010). Although it is not known how strand specificity is achieved in C. elegans or how important star miRNAs are in any organisms, the machinery producing distinct age-depen- 
dent accumulation patterns of mature/star strands, if any, will be an important issue since it would potentially affect aging through strand selection and/or by stability of mature/ star strands.

In terms of miRNA stability, it is also worth investigating how miRNAs are negatively regulated during aging. Here we examined changes in expression of miRNAs in temperature-shift-induced modified lifespan backgrounds and found that miRNAs with decreased expression during aging rapidly reduced their levels in both normal and long-lived conditions. Also, we previously found some miRNAs that changed their expression dynamically during development (e.g., miR-795, one of the let-7 family miRNAs, which has its peak expression in the L3 [the third larval stage] and then is quickly down-regulated toward the young adult stage) (Kato et al. 2009a). These observations imply a mechanism that positively reduces levels of mature miRNAs, potentially conducted by an exoribonuclease like XRN-2 (Chatterjee and Grosshans 2009) or miRNA star strands, which have a nearly perfect base-pairing match with partner mature strands, like anti-miRNAs (antisense-miRNA oligonucleotides) (Krutzfeldt et al. 2005).

In addition to miRNA star strands, we identified additional novel miRNA candidates in our cDNA libraries for small RNAs. Their expression levels were mostly quite low, but we found that some of them were expressed in neurons, including sensory neurons in the head (e.g., ADLs), suggesting their possible role in the response to environmental stimuli. Although for two of the novel miRNA candidates, it remains unclear whether they are real miRNAs or not, since their expression levels were not affected in either alg-1(gk214) or alg-2(ok304) mutant backgrounds, they are still interesting candidates to pursue since both of them showed a decrease in expression during aging. Also, our validation approach using Argonaute mutants may reveal an unexpected functional complexity in Argonaute proteins. Both C. elegans ALG-1 and ALG-2 are members of the highly conserved RDE-1/AGO1/PIWI family of proteins that regulate post-transcriptional gene silencing, and it is thought that ALG-1 functions as the primary miRNP, and ALG-2 acts redundantly with ALG-1 to support it. This is based on the observation that alg-1 (gk214) mutants cause a vulval phenotype, resulting in premature death at the young adult stage, while alg-2(ok304) mutants are grossly normal. Our study of novel miRNA expression in alg-1/2 mutants suggests that some of the miRNAs depend on both alg-1 and alg-2, and this dependency might vary during development and possibly during aging as well. Also, we noticed that one of the novel miRNA candidates that showed a dramatic reduction in expression in the alg-1(gk214) mutant background (1277767_adh in Fig. 8D) maps to a tranposase-coding region, meaning that expression of a transposase gene-derived small RNA depends on Argonaute protein activity. This observation might suggest the possibility that, like siRNAs, $21 \mathrm{U}-\mathrm{RNAs} / \mathrm{piRNAs}$, or $22 \mathrm{G}-$
RNAs (Sijen and Plasterk 2003; Batista et al. 2008; Gu et al. 2009), transposon-derived miRNAs also contribute to genome stability through the control of transposon activity.

In addition to new classes of small noncoding RNAs, we found an interesting age-associated feature in the known, classical noncoding RNAs, such as tRNAs and snoRNAs; cleaved RNA fragments of these noncoding RNAs accumulated during aging. These, especially tRNA-derived shorter fragments, may have the potential to modulate aging and to connect aging and the stress response since it has been shown that tRNA cleavage is induced in response to stress in several organisms (Thompson and Parker 2009a). We, indeed, found a dramatic increase in the accumulation of tRNA cleavage products in C. elegans that were exposed to stress such as heat shock (M Kato and FJ Slack, unpubl.). This suggests that stress-induced cleavage of tRNAs is a conserved, fundamental stress response. Although their biological function remains largely unclear, some studies have shown that tRNA-derived fragments were co-purified with an Argonaute protein complex (Kawamura et al. 2008), suggesting that such tRNA fragments could potentially function like siRNAs or miRNAs and regulate the translational machinery. Indeed, in the case of snoRNAs, recent reports suggest that snoRNA-derived small RNAs can function like miRNAs (Ender et al. 2008; Taft et al. 2009). We also found a significant number of snoRNA-derived short reads in our libraries which showed an increase in accumulation with age, and indeed, some mature snoRNAs appear to resemble miRNA hairpin-loop precursors in their secondary structures (data not shown).

The novel sncRNAs we report in this study, including cleaved RNA fragments, might also mediate sequencespecific regulation like miRNAs. Additional study of the potential targets interacting with these small RNAs might open up a new field in small RNA-related biology. Also, several observations suggest that stress-induced RNA cleavage and induction of ribonucleases may be involved in cancer and other diseases (Thompson and Parker 2009a). Further analysis of small noncoding RNA expression profiles and detailed genetic studies will give new insights into novel molecular networks in aging and common mechanisms in aging and age-related diseases.

\section{MATERIALS AND METHODS}

\section{C. elegans strains}

The spe-9(hc88) strain was first obtained from CGC (Caenorhabditis Genetic Center) and then backcrossed nine times to our standard wild-type N2 lab strain. Animals were maintained for a few generations without starvation at the permissive temperature, $15^{\circ} \mathrm{C}$, to collect embryos. After embryos were harvested, they were incubated at $23^{\circ} \mathrm{C}$ in $\mathrm{M} 9$ buffer without a food source overnight to arrest their growth at the early L1 (the first larval) stage. Synchronized animals were transferred to bacteria-seeded plates 
and cultured at $23^{\circ} \mathrm{C}$ to induce their sterility. The aging stages used for RNA preparation were Day 0, Day 5, Day 8, and Day 12 post-L4 molt, or as shown in each experiment. Day 0 in this study represents the day of the final molt, $\sim 48 \mathrm{~h}$ after staged $\mathrm{L} 1$ animals were placed on seeded plates at $23^{\circ} \mathrm{C}$. In alg-1 RNAi and temperature-shift experiments, animals were treated with each condition after Day 0 (young adult stage) of adulthood. The two mutant strains, alg-1(gk214) and alg-2(ok304), were obtained from the CGC and maintained at $15^{\circ} \mathrm{C}$ similar to the spe-9(hc88) strain, but they were cultured at $15^{\circ} \mathrm{C}$ during all stages since alg-1(gk214) mutants are sick and show a vulval bursting phenotype at a higher temperature. Total RNAs were purified from alg-1/2 mutants $72 \mathrm{~h}$ and $84 \mathrm{~h}$ after synchronized L1 stage animals were fed, for L4 and young adult stages, respectively. For miRNA promoter::gfp fusion lines, details are shown in Supplemental Figure S6.

\section{Lifespan assays}

Lifespan assays were basically conducted as previously described (Boehm and Slack 2005), except that FUDR (5-fluorodeoxyuridine, a DNA replication inhibitor) was not used in this study. Similar to those used for RNA isolation mentioned above, synchronized Day 0 young adult animals were transferred into different conditions (e.g., $15^{\circ} \mathrm{C}$ and on RNAi bacteria). Survival of animals was examined every day. Animals that crawled off the plate or burst were excluded from the calculations. Approximately 100 animals were tested on each plate with 2-3 replicates, and at least two independent assays were performed for each result.

\section{RNA, cDNA library preparation and Solexa deep-sequencing}

spe-9(hc88) animals were first washed with M9 buffer multiple times in order to remove bacteria. Then, RNAs were purified using the mirVana miRNA Isolation Kit (Ambion), according to the manufacturer's instructions. In the initial deep-sequencing experiment, cDNA libraries were made from $10 \mu \mathrm{g}$ of RNAs enriched from a small RNA fraction (<200 nt), which were prepared based on the manufacturer's instructions in the mirVana kit. In the second deep-sequencing experiment (verification sets from Day 0 and Day 8), we used $10 \mu \mathrm{g}$ of total RNA for constructing cDNA libraries. Since our sequencing results were essentially identical, we now confirm that either procedure leads to consistent results. Preparation of cDNA libraries for the Solexa deep-sequencing experiment was carried out using the DGE-Small RNA Sample Prep Kit ver. 1.0 (Illumina) according to the manufacturer's instructions. Specifically, RNAs corresponding to 15-30 nt in size were selectively purified, and then these were ligated to adapters and amplified by RT-PCR. The same amount of purified library DNA was captured on an Illumina flow cell for cluster generation and was sequenced for 36 cycles on an Illumina Genome Analyzer II following the manufacturer's protocols for single-end reads (DGE-Small RNA Cluster Generation Kit and 36 Cycle Solexa Sequencing Kit). All our raw deep-sequencing data and processed data are available from the GEO database (GSE18634).

\section{Quantitative RT-PCR}

We used qRT-PCR with TaqMan Small RNA Assays (Applied Biosystems) for confirmation of the deep-sequencing results and for examining changes in expression of miRNAs in alg-1 RNAi- induced abnormal lifespan background, in different environmental conditions and in the alg-1/2 mutant background, according to the manufacturer's instructions. For validating expression of novel miRNA candidates in the alg-1/2 mutant background, the results were normalized to the expression level of U18, which is widely used as a control in TaqMan qRT-PCR. For testing miRNA expression during aging, we used the expression of act-3 and ama-1 using TaqMan Gene Expression Assay (Applied Biosystems) as controls, both of which are thought to be constantly expressed in aging (Evans et al. 2008; Fuhrman et al. 2009). Note that the results were normalized with the "average" of these multiple stably expressed reference genes since there is no universally accepted gene for normalization during aging (Nolan et al. 2006). Also, we used 50-100 ng/ $\mu \mathrm{l}$ of total RNAs for novel miRNA candidates and 10 $\mathrm{ng} / \mu \mathrm{l}$ for known miRNAs and for controls (e.g., U18) since the level of expression of novel miRNA candidates was much lower. The results were analyzed by the delta-delta Ct method, and $P$-values were calculated by $t$-test from delta $\mathrm{Ct}$ values of the control and the target.

\section{Microscopic study of miRNA promoter::gfp transgenic lines}

Age-related changes in GFP signals expressed from miRNA promoter::gfp lines were examined by microscopic observation. All strains used were crossed into the spe-9(hc88) background. To obtain images from a whole animal body, an individual animal was exposed to a UV light for the same exposure time and with a focus on the center of each animal based on its pharynx and/or vulva. Since signals obtained with a longpass GFP (LPGFP) filter contain both those from intestinal cellular autofluorescence and transgene-expressing GFP, signals obtained with a TRITC filter that only reflects age-related autofluorescence were subtracted from the signals obtained with a LPGFP filter after normalizing the differences of signal levels in LPGFP and TRITC filters based on those detected in non-GFP spe-9(hc88) animals at each time point and each condition. Signal intensities were calculated using the NIH ImageJ program (http://rsbweb.nih.gov/ij/) from $\sim 15-20$ animals at each time point of each line.

\section{Computational sequence data analysis}

We required perfect sequence matching from the processed, aligned reads to assess the number of miRNA and other small RNA reads in each sample if not specified in each computational analysis. The raw data were aligned to the C. elegans genome using the SOAP program (ver. 1.10) (Li et al. 2008) with the following command options: $-\mathrm{w} 5-\mathrm{S} 3-\mathrm{A}$ (the $3^{\prime}$ adapter sequence used) (maximum two base pair mismatches were allowed as a default option). We controlled library differences by normalizing to the total number of reads that matched the C. elegans genome (WormBase WS190) in each sample. The number of sequence reads in each sample was finally standardized to the Day 0 sample in each set of deep-sequencing experiments. The data sets of known miRNAs and 21U-RNAs were obtained from miRBase (Release 14.0) and WormBase (WS215), respectively.

For testing the proportion of each noncoding RNA species in each sample, the number of reads with perfect matches was counted after searching with the blastn program (ver. 2.2.17). The parameters in the blastn program we used are as follows: -e $0.001-\mathrm{G} 5$-E 
2 -q -3 -r 1 -W 7 -v 10 -b 10. The miRDeep program was used for finding novel miRNA candidates by basically following the default procedure provided with the program (Friedlander et al. 2008), and the RNAfold program (Vienna RNA package ver. 1.6.5; http:// www.tbi.univie.ac.at/ ivo/RNA/) was used for predicting secondary structure of primary miRNA transcripts and snoRNAs. All genome coordinates shown in this study are based on WormBase genome build WS190.

For clustering analysis of miRNAs, we used the Cluster 3.0 program (uncentered correlation option with normalization) (Eisen et al. 1998), and the Java TreeView program (Saldanha 2004) was then used to visualize results.

Deep-sequencing data obtained from the GEO database are as follows: GSE20649, Argonaute proteins ChIP-Seq libraries, was used for the analysis of mature and star miRNA species; GSE11735, prg-1 mutant, was used for the analysis of 21nt-U-RNAs; GSE18231, mutants in WAGO pathway, and GSE18165, mutants in CSR-1 pathway, were used for the analysis of 26nt-G-RNAs and 22nt-G-RNAs.

\section{Statistical analysis of deep-sequencing data}

To identify differentially expressed miRNAs, pairwised hypothesis testings for Day 0, Day 5, Day 8, and Day 12 were used. Since we did not have a complete set of experimental replicates, we took advantage of two replicates of Day 0 and Day 8. Linear regressions on mean $(\mu)$ and variance $\left(\sigma^{2}\right)$ of 174 miRNAs showed that $\sigma^{2}=$ $\mu^{1.7}$ for both Day 0 and Day 8. Considering that expressions of different days should have similar distribution patterns (biases and errors are caused by technical processes), we assumed that all days follow the normal distribution of $\mathrm{N}\left(\mu, \mu^{1.7}\right)$. Based on the distribution, we used the $t$-test to calculate the pairwise $P$-values of all four stages of aging samples. The final $P$-value for each miRNA is the minimum $P$-value among the six pairwise comparisons. To confirm our selection, another method was applied to our data set. We calculated a time-course $P$-value across all days, based on the chi-square distribution after variance stabilizing transformation. Since variance is a power function of mean $\left(\sigma^{2}=\mu^{1.7}\right)$, we wanted a transformation of data that had constant variance. Based on a first-order Taylor series expansion at point $\mu$ and supposing $\sigma^{2}=\alpha^{2} \mu^{2 \beta}$, we have

$$
f(x)=\frac{1}{\alpha}\left[\frac{x^{-\beta+1}}{-\beta+1}\right],
$$

where $\alpha=1$ and $\beta=0.85$. After the transformation of data, $P$-values across all days were calculated based on a chi-square distribution with three degrees of freedom. All differentially expressed miRNAs identified by the time-course method could also be identified by the pairwise comparison.

\section{Computational target prediction of age-associated miRNAs and pathway analysis}

To identify the potential targets of age-associated miRNAs, we combined analysis of the computational target prediction and gene expression microarray data. First, miRanda (http://www. microrna.org/microrna/home.do) (John et al. 2004), TargetScan (http://www.targetscan.org/) (Lewis et al. 2005), and PicTar (http:// www.pictar.org/) (Lall et al. 2006) were used to predict the targets based on seed matching, cross-species conservation, and targeted pair energy. Then, to reduce the false positive targets in the prediction, we incorporated the microarray data (Table S3 in Budovskaya et al. 2008), which have expression levels of protein-coding genes during aging in C. elegans at the same time points as our small RNA study. Based on the fact that miRNAs or co-expressed miRNAs tend to target the genes from the same functional categories or pathways, we used gene set enrichment analysis to identify differentially expressed genes. Pathways information was obtained from KEGG (Kyoto Encyclopedia of Genes and Genomes; http://www.genome.jp/kegg/.). Then, Fisher's exact test was used to find significant pathways. If a gene predicted by one of the prediction programs mentioned above is also present in the significant pathways, this gene is considered to be the target of the corresponding miRNAs. The reduced target list is subjected to gene set enrichment analysis (by Fisher's exact test) in order to see if any interesting pathways are enriched in our predicted targets. A list of genes involved in or necessary for the normal aging process is obtained from "Human Ageing Genomic Resources" (http:// genomics.senescence.info/genes/models.html) and based on the GO Term "determination of adult life span [GO:0008340]."

\section{DATA DEPOSITION}

Deep-sequencing data from this study has been submitted to the GEO database: GSE18634.

\section{SUPPLEMENTAL MATERIAL}

Supplemental material is available for this article.

\section{ACKNOWLEDGMENTS}

We thank Ghia Euskirchen and Hannah Monahan for help with Solexa sequencing, and Zachary Pincus and Alexandre de Lencastre for critical reading of this manuscript. We also thank Zhi John Lu, Mike Wilson, and Nicole Washington for handling of the deepsequencing data, and the CGC for strains. We also thank Yale University Biomedical High Performance Computing Center and NIH grant RR19895, which funded the instrumentation. M.K. was partially supported by a postdoctoral fellowship from the Uehara Life Science Foundation; X.C. was supported by a fellowship from the China Scholarship Council; F.S. was supported by grants from the NIH to the modENCODE consortium (RFAHG-06-006) and R01 AG033921 and from the Ellison Medical Foundation.

Received March 10, 2011; accepted June 24, 2011.

\section{REFERENCES}

Antebi A. 2007. Genetics of aging in Caenorhabditis elegans. PLoS Genet 3: 1565-1571.

Bagga S, Bracht J, Hunter S, Massirer K, Holtz J, Eachus R, Pasquinelli AE. 2005. Regulation by let-7 and lin-4 miRNAs results in target mRNA degradation. Cell 122: 553-563.

Batista PJ, Ruby JG, Claycomb JM, Chiang R, Fahlgren N, Kasschau KD, Chaves DA, Gu W, Vasale JJ, Duan S, et al. 2008. PRG-1 and 
21U-RNAs interact to form the piRNA complex required for fertility in C. elegans. Mol Cell 31: 67-78.

Boehm M, Slack F. 2005. A developmental timing microRNA and its target regulate life span in C. elegans. Science 310: 1954-1957.

Budovskaya YV, Wu K, Southworth LK, Jiang M, Tedesco P, Johnson TE, Kim SK. 2008. An elt-3/elt-5/elt-6 GATA transcription circuit guides aging in C. elegans. Cell 134: 291-303.

Chatterjee S, Grosshans H. 2009. Active turnover modulates mature microRNA activity in Caenorhabditis elegans. Nature 461: 546-549.

Claycomb JM, Batista PJ, Pang KM, Gu W, Vasale JJ, van Wolfswinkel JC, Chaves DA, Shirayama M, Mitani S, Ketting RF, et al. 2009. The Argonaute CSR-1 and its 22G-RNA cofactors are required for holocentric chromosome segregation. Cell 139: 123-134.

Corrêa RL, Steiner FA, Berezikov E, Ketting RF. 2010. MicroRNAdirected siRNA biogenesis in Caenorhabditis elegans. PLoS Genet 6: e1000903. doi: 10.1371/journal.pgen.1000903.

Czech B, Zhou R, Erlich Y, Brennecke J, Binari R, Villalta C, Gordon A, Perrimon N, Hannon GJ. 2009. Hierarchical rules for Argonaute loading in Drosophila. Mol Cell 36: 445-456.

de Lencastre A, Pincus Z, Zhou K, Kato M, Lee SS, Slack FJ. 2010. MicroRNAs both promote and antagonize longevity in C. elegans. Curr Biol 20: 2159-2168.

Eisen MB, Spellman PT, Brown PO, Botstein D. 1998. Cluster analysis and display of genome-wide expression patterns. Proc Natl Acad Sci 95: 14863-14868.

Ender C, Krek A, Friedlander MR, Beitzinger M, Weinmann L, Chen W, Pfeffer S, Rajewsky N, Meister G. 2008. A human snoRNA with microRNA-like functions. Mol Cell 32: 519-528.

Evans EA, Chen WC, Tan MW. 2008. The DAF-2 insulin-like signaling pathway independently regulates aging and immunity in C. elegans. Aging Cell 7: 879-893.

Fabian TJ, Johnson TE. 1994. Production of age-synchronous mass cultures of Caenorhabditis elegans. J Gerontol 49: B145-B156.

Friedlander MR, Chen W, Adamidi C, Maaskola J, Einspanier R, Knespel S, Rajewsky N. 2008. Discovering microRNAs from deep sequencing data using miRDeep. Nat Biotechnol 26: 407-415.

Friedman DB, Johnson TE. 1988. Three mutants that extend both mean and maximum life span of the nematode, Caenorhabditis elegans, define the age-1 gene. J Gerontol 43: B102-B109.

Fuhrman LE, Goel AK, Smith J, Shianna KV, Aballay A. 2009. Nucleolar proteins suppress Caenorhabditis elegans innate immunity by inhibiting p53/CEP-1. PLoS Genet 5: e1000657. doi: 10.1371/journal.pgen.1000657.

Garigan D, Hsu AL, Fraser AG, Kamath RS, Ahringer J, Kenyon C. 2002. Genetic analysis of tissue aging in Caenorhabditis elegans: A role for heat-shock factor and bacterial proliferation. Genetics 161: 1101-1112.

Gent JI, Lamm AT, Pavelec DM, Maniar JM, Parameswaran P, Tao L, Kennedy S, Fire AZ. 2010. Distinct phases of siRNA synthesis in an endogenous RNAi pathway in C. elegans soma. Mol Cell 37: 679689.

Ghildiyal M, Xu J, Seitz H, Weng Z, Zamore PD. 2010. Sorting of Drosophila small silencing RNAs partitions microRNA* strands into the RNA interference pathway. RNA 16: 43-56.

Golden TR, Melov S. 2004. Microarray analysis of gene expression with age in individual nematodes. Aging Cell 3: 111-124.

Grishok A, Pasquinelli AE, Conte D, Li N, Parrish S, Ha I, Baillie DL, Fire A, Ruvkun G, Mello CC. 2001. Genes and mechanisms related to RNA interference regulate expression of the small temporal RNAs that control C. elegans developmental timing. Cell 106: 23-34.

Gu W, Shirayama M, Conte D Jr, Vasale J, Batista PJ, Claycomb JM, Moresco JJ, Youngman EM, Keys J, Stoltz MJ, et al. 2009. Distinct argonaute-mediated 22G-RNA pathways direct genome surveillance in the C. elegans germline. Mol Cell 36: 231-244.

Guo H, Ingolia NT, Weissman JS, Bartel DP. 2010. Mammalian microRNAs predominantly act to decrease target mRNA levels. Nature 466: 835-840.

Haigis MC, Yankner BA. 2010. The aging stress response. Mol Cell 40: 333-344.
He L, He X, Lowe SW, Hannon GJ. 2007. MicroRNAs join the p53 network-another piece in the tumour-suppression puzzle. Nat Rev Cancer 7: 819-822.

Herndon LA, Schmeissner PJ, Dudaronek JM, Brown PA, Listner KM, Sakano Y, Paupard MC, Hall DH, Driscoll M. 2002. Stochastic and genetic factors influence tissue-specific decline in aging C. elegans. Nature 419: 808-814.

Ibanez-Ventoso C, Yang M, Guo S, Robins H, Padgett RW, Driscoll M. 2006. Modulated microRNA expression during adult lifespan in Caenorhabditis elegans. Aging Cell 5: 235-246.

John B, Enright AJ, Aravin A, Tuschl T, Sander C, Marks DS. 2004. Human microRNA targets. PLoS Biol 2: e363. doi: 10.1371/ journal.pbio.0020363.

Johnson CD, Esquela-Kerscher A, Stefani G, Byrom M, Kelnar K, Ovcharenko D, Wilson M, Wang X, Shelton J, Shingara J, et al. 2007. The let-7 microRNA represses cell proliferation pathways in human cells. Cancer Res 67: 7713-7722.

Johnston J, Iser WB, Chow DK, Goldberg IG, Wolkow CA. 2008. Quantitative image analysis reveals distinct structural transitions during aging in Caenorhabditis elegans tissues. PLoS ONE 3: e2821. doi: 10.1371/journal.pone.0002821.

Kanehisa M, Goto S, Furumichi M, Tanabe M, Hirakawa M. 2010. KEGG for representation and analysis of molecular networks involving diseases and drugs. Nucleic Acids Res 38: D355-D360.

Kato M, de Lencastre A, Pincus Z, Slack FJ. 2009a. Dynamic expression of small noncoding RNAs, including novel microRNAs and piRNAs/21U-RNAs, during Caenorhabditis elegans development. Genome Biol 10: R54. doi: 10.1186/gb-2009-10-5-r54.

Kato M, Paranjape T, Muller RU, Nallur S, Gillespie E, Keane K, Esquela-Kerscher A, Weidhaas JB, Slack FJ. 2009b. The mir-34 microRNA is required for the DNA damage response in vivo in $C$. elegans and in vitro in human breast cancer cells. Oncogene 28: 2419-2424.

Kawamura Y, Saito K, Kin T, Ono Y, Asai K, Sunohara T, Okada TN, Siomi MC, Siomi H. 2008. Drosophila endogenous small RNAs bind to Argonaute 2 in somatic cells. Nature 453: 793-797.

Kenyon CJ. 2010. The genetics of aging. Nature 464: 504-512.

Kenyon C, Chang J, Gensch E, Rudner A, Tabtiang R. 1993. A C. elegans mutant that lives twice as long as wild type. Nature 366: 461-464.

Klass MR. 1977. Aging in the nematode Caenorhabditis elegans: Major biological and environmental factors influencing life span. Mech Ageing Dev 6: 413-429.

Klass MR. 1983. A method for the isolation of longevity mutants in the nematode Caenorhabditis elegans and initial results. Mech Ageing Dev 22: 279-286.

Kozomara A, Griffiths-Jones S. 2011. miRBase: Integrating microRNA annotation and deep-sequencing data. Nucleic Acids Res 39: D152D157.

Krutzfeldt J, Rajewsky N, Braich R, Rajeev KG, Tuschl T, Manoharan M, Stoffel M. 2005. Silencing of microRNAs in vivo with "antagomirs". Nature 438: 685-689.

Lall S, Grun D, Krek A, Chen K, Wang YL, Dewey CN, Sood P, Colombo T, Bray N, Macmenamin P, et al. 2006. A genome-wide map of conserved microRNA targets in C. elegans. Curr Biol 16: 460-471.

Lee RC, Feinbaum RL, Ambros V. 1993. The C. elegans heterochronic gene lin-4 encodes small RNAs with antisense complementarity to lin-14. Cell 75: 843-854.

Lewis BP, Burge CB, Bartel DP. 2005. Conserved seed pairing, often flanked by adenosines, indicates that thousands of human genes are microRNA targets. Cell 120: 15-20.

Li R, Li Y, Kristiansen K, Wang J. 2008. SOAP: Short oligonucleotide alignment program. Bioinformatics 24: 713-714.

Lim LP, Lau NC, Weinstein EG, Abdelhakim A, Yekta S, Rhoades MW, Burge CB, Bartel DP. 2003. The microRNAs of Caenorhabditis elegans. Genes Dev 17: 991-1008.

Lund J, Tedesco P, Duke K, Wang J, Kim SK, Johnson TE. 2002. Transcriptional profile of aging in C. elegans. Curr Biol 12: 1566 1573. 
Mattout A, Dechat T, Adam SA, Goldman RD, Gruenbaum Y. 2006. Nuclear lamins, diseases, and aging. Curr Opin Cell Biol 18: 335-341.

McCarroll SA, Murphy CT, Zou S, Pletcher SD, Chin CS, Jan YN, Kenyon C, Bargmann CI, Li H. 2004. Comparing genomic expression patterns across species identifies shared transcriptional profile in aging. Nat Genet 36: 197-204.

Morimoto RI. 2008. Proteotoxic stress and inducible chaperone networks in neurodegenerative disease and aging. Genes Dev 22: 1427-1438.

Morris JZ, Tissenbaum HA, Ruvkun G. 1996. A phosphatidylinositol3 -OH kinase family member regulating longevity and diapause in Caenorhabditis elegans. Nature 382: 536-539.

Nolan T, Hands RE, Bustin SA. 2006. Quantification of mRNA using real-time RT-PCR. Nat Protoc 1: 1559-1582.

Okamura K, Liu N, Lai EC. 2009. Distinct mechanisms for microRNA strand selection by Drosophila Argonautes. Mol Cell 36: 431-444.

Olsen A, Vantipalli MC, Lithgow GJ. 2006. Checkpoint proteins control survival of the postmitotic cells in Caenorhabditis elegans. Science 312: 1381-1385.

Pak J, Fire A. 2007. Distinct populations of primary and secondary effectors during RNAi in C. elegans. Science 315: 241-244.

Roush S, Slack FJ. 2008. The let-7 family of microRNAs. Trends Cell Biol 18: 505-516.

Ruby JG, Jan C, Player C, Axtell MJ, Lee W, Nusbaum C, Ge H, Bartel DP. 2006. Large-scale sequencing reveals 21U-RNAs and additional microRNAs and endogenous siRNAs in C. elegans. Cell 127: $1193-1207$.

Saldanha AJ. 2004. Java Treeview-extensible visualization of microarray data. Bioinformatics 20: 3246-3248.

Schaefer M, Pollex T, Hanna K, Tuorto F, Meusburger M, Helm M, Lyko F. 2010. RNA methylation by Dnmt2 protects transfer RNAs against stress-induced cleavage. Genes Dev 24: 1590-1595.

Sijen T, Plasterk RH. 2003. Transposon silencing in the Caenorhabditis elegans germ line by natural RNAi. Nature 426: 310-314.
Singson A, Mercer KB, L'Hernault SW. 1998. The C. elegans spe-9 gene encodes a sperm transmembrane protein that contains EGFlike repeats and is required for fertilization. Cell 93: 71-79.

Stefani G, Slack FJ. 2008. Small noncoding RNAs in animal development. Nat Rev Mol Cell Biol 9: 219-230.

Stoeckius M, Maaskola J, Colombo T, Rahn HP, Friedlander MR, Li N, Chen W, Piano F, Rajewsky N. 2009. Large-scale sorting of $C$. elegans embryos reveals the dynamics of small RNA expression. Nat Methods 6: 745-751.

Tabara H, Sarkissian M, Kelly WG, Fleenor J, Grishok A, Timmons L, Fire A, Mello CC. 1999. The rde-1 gene, RNA interference, and transposon silencing in C. elegans. Cell 99: 123-132.

Taft RJ, Glazov EA, Lassmann T, Hayashizaki Y, Carninci P, Mattick JS. 2009. Small RNAs derived from snoRNAs. RNA 15: 1233-1240.

Thompson DM, Parker R. 2009a. Stressing out over tRNA cleavage. Cell 138: 215-219.

Thompson DM, Parker R. 2009b. The RNase Rnylp cleaves tRNAs and promotes cell death during oxidative stress in Saccharomyces cerevisiae. J Cell Biol 185: 43-50.

Tops BB, Plasterk RH, Ketting RF. 2006. The Caenorhabditis elegans Argonautes ALG-1 and ALG-2: Almost identical yet different. Cold Spring Harb Symp Quant Biol 71: 189-194.

Vella MC, Choi EY, Lin SY, Reinert K, Slack FJ. 2004. The C. elegans microRNA let-7 binds to imperfect let-7 complementary sites from the lin-41 3'UTR. Genes Dev 18: 132-137.

Wallace DC. 2005. A mitochondrial paradigm of metabolic and degenerative diseases, aging, and cancer: A dawn for evolutionary medicine. Annu Rev Genet 39: 359-407.

Yamasaki S, Ivanov P, Hu GF, Anderson P. 2009. Angiogenin cleaves tRNA and promotes stress-induced translational repression. J Cell Biol 185: 35-42.

Yang JS, Phillips MD, Betel D, Mu P, Ventura A, Siepel AC, Chen KC, Lai EC. 2010. Widespread regulatory activity of vertebrate microRNA* species. RNA 17: 312-326. 

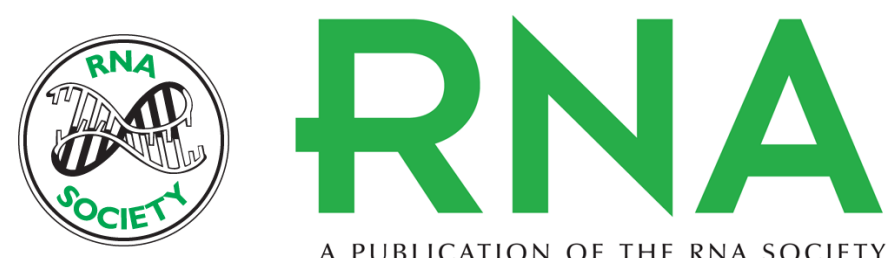

A PUBLICATION OF THE RNA SOCIETY

\section{Age-associated changes in expression of small, noncoding RNAs, including microRNAs, in C. elegans}

Masaomi Kato, Xiaowei Chen, Sachi Inukai, et al.

RNA 2011 17: 1804-1820 originally published online August 2, 2011

Access the most recent version at doi:10.1261/rna.2714411

\section{Supplemental http://rnajournal.cshlp.org/content/suppl/2011/07/22/rna.2714411.DC1 \\ Material}

References This article cites 72 articles, 14 of which can be accessed free at:

http://rnajournal.cshlp.org/content/17/10/1804.full.html\#ref-list-1

\section{License}

Email Alerting Receive free email alerts when new articles cite this article - sign up in the box at the Service top right corner of the article or click here.

To subscribe to RNA go to:

http://rnajournal.cshlp.org/subscriptions 